\title{
Domain theory and differential calculus (functions of one variable)
}

\author{
ABBAS EDALAT $T^{\dagger}$ and ANDRÉ LIEUTIER \\ ${ }^{\dagger}$ Department of Computing, Imperial College, London, UK \\ Email: ae@doc.ic.ac.uk \\ ${ }^{\ddagger}$ Dassault Systemes Provence and LMC/IMAG, Aix-en-Provence and Grenoble, France
}

Received 2 September 2002; revised 20 November 2003

We introduce a domain-theoretic framework for differential calculus. We define the set of primitive maps as well as the derivative of an interval-valued Scott continuous function on the domain of intervals, and show that they are dually related, providing an extension of the classical duality of differentiation and integration as in the fundamental theorem of calculus. It is shown that, for locally Lipschitz functions of a real variable, the domain-theoretic derivative coincides with the Clarke's derivative. We then construct a domain for differentiable real-valued functions of a real variable by pairing consistent information about the function and information about its derivative. The set of classical $C^{1}$ functions, equipped with its $C^{1}$ norm, is embedded into the set of maximal elements of this countably based, bounded complete continuous domain. This domain also provides a model for the differential properties of piecewise $C^{1}$ functions, locally Lipschitz functions and more generally of all continuous functions. We prove that consistency of function information and derivative information is decidable on rational step functions, which shows that our domain can be given an effective structure. We thus obtain a data type for differential calculus. As an immediate application, we present a domain-theoretic formulation of Picard's theorem, which provides a data type for solving differential equations.

\section{Introduction}

We introduce a domain-theoretic framework for differentiable functions, which leads to a data type for differentiable functions and a data type for solving differential equations. Differential calculus, introduced by Newton and Leibnitz in the 17th century, has provided the foundation of modern science and technology, and is the basis of applied mathematics, mathematical physics and scientific computation. By constructing data types for differential calculus, we seek to bring smooth mathematics into the realm of computer science.

The key element in our framework is the notion of the set of primitive maps of a Scott continuous function on the domain of intervals, which is derived here from basic principles. First, the concept of an interval Lipschitz constant of a Scott continuous function is introduced. A non-trivial interval Lipschitz constant of a classical continuous function in an open interval is given by a compact interval whose left and right end points are, respectively, a lower and upper Lipschitz constant for the function in that open interval. An interval Lipschitz constant in an open interval thus corresponds to a single-step function, 
and the collection of interval Lipschitz constants is in bijective correspondence with the collection of single-step functions. The natural concept of interval Lipschitz constant in an open interval, and its corresponding single-step function, can be generalised to Scott continuous functions of the domain of intervals. The set of primitive maps of a single-step function is then defined as the collection, or the single-step tie, of all Scott continuous functions that have interval Lipschitz constants corresponding to the singlestep function. Using the fact that any Scott continuous function can be constructed from single-step functions, the set of primitive maps of a Scott continuous function can then be defined as a collection, or tie, of Scott continuous functions with interval Lipschitz constants represented by the integrand. The collection of ties ordered by reverse inclusion is a directed complete partial order and the primitive sets function, which for a Scott continuous interval-valued function outputs its corresponding tie of Scott continuous functions, is itself Scott continuous.

An interval Lipschitz constant of a Scott continuous function gives local information on the differential properties of the function. By collecting all such information, we move from a local to a global viewpoint and define the domain-theoretic derivative of a Scott continuous function on the domain of intervals, which is itself shown to be a Scott continuous function. This is in sharp contrast to the situation in classical analysis in which a continuous function may not have a derivative at a point or indeed at any point at all. In the domain-theoretic world, such irregularities do not occur and the derivative of any Scott continuous function is itself a function of the same type.

We derive a duality between the domain-theoretic derivative and the primitive maps function, which can be considered as an interval version of the fundamental theorem of calculus for interval-valued functions of an interval variable: a Scott continuous function $f$ is a primitive map of a Scott continuous function $g$ if and only if the domain-theoretic derivative of $f$ refines $g$. In the case of interval-valued functions of a real variable this duality is closely linked with the fundamental theorem of classical calculus.

When we restrict to functions with real input, the domain-theoretic derivative of a locally Lipschitz function of one real variable coincides with the so-called Clarke's gradient (Clarke 1983; Clarke et al. 1998). For functions of $n$ real variables as treated in Edalat et al. (2004), which is the sequel to the present paper, the domain-theoretic derivative of a locally Lipschitz function at a point gives the smallest $n$-dimensional rectangle, with sides parallel to the coordinate axis, that contains the Clarke's gradient at that point. The domain-theoretic derivative, however, extends to functions of an interval variable, as we will first consider in this paper.

The central part of this work is the construction of a domain $D^{1}$ for differentiable real-valued functions of a real variable. This domain is a subset of the product of two copies of the domain of Scott continuous interval-valued functions on the domain of intervals, where the subset is characterised by a consistency relation between two Scott continuous functions. In a consistent pair of functions, the first function represents an approximation to the $C^{1}$ function itself, while the second function in the pair gives an approximation to the derivative of this $C^{1}$ function. The consistency relation is Scott closed and decidable on the basis consisting of pairs of rational step functions, which shows that $D^{1}$ is a countably based bounded complete continuous domain that can be 
given an effective structure. The set of classical $C^{1}$ functions, equipped with its $C^{1}$ norm, is embedded into the set of maximal elements of $D^{1}$. Furthermore, the set of piecewise $C^{1}$ functions, the set of locally Lipschitz functions and the set of all continuous functions are each embedded into the set of maximal elements of $D^{1}$, thereby giving a model for the differential properties of various types of functions.

As an immediate application, we present a domain-theoretic and effective generalisation of Picard's theorem, which provides a data type and an algorithm for solving differential equations given by a vector field and an initial condition. At each step of computation of this algorithm, one gets an approximation that is an interval piecewise polynomial function with rational coefficients that provides information about the solution, even when both the initial condition and the specifying vector field are given with uncertainty.

Another application of this work will be to provide an effective version of what is referred to as the implicit function theorem, which is a main tool in the study of manifolds. In the one variable case considered in this paper, the effective version of the implicit function theorem provides an algorithm to obtain the isolated roots of differentiable functions. In multi-variate differential calculus, which will be treated in a future paper, the implicit function theorem is used extensively in the geometric design of smooth curves and surfaces. Since the functions defining the curves or surfaces used in CAD software can be of various concrete classes (piecewise polynomial, rational, trigonometric as well as more 'exotic' classes of smooth functions), the use of abstract classes representing $C^{k}$ functions allows us to design generic algorithms for solving systems of equations arising, for example, in surface intersections, ray shooting, and many other geometric operators. In fact, the data type introduced here can provide a sound mathematical framework on top of which these existing practices could be founded (Hu et al. 1996; Sakkalis et al. 2001).

The present work is the continuation of a 10 year old project for constructing domaintheoretic computational models in various subjects including fractal geometry, measure and integration, real number computation, computational geometry and solid modelling (Edalat 1995b; Edalat 1995a; Lawson 1997; Edalat and Heckmann 1998; Escardó 1996; Edalat and Escardó 2000; Edalat and Potts 1997; Edalat and Sünderhauf 1998; Edalat 1997; Edalat and Lieutier 2002). Although the question of computability for differentiable functions and their derivatives has been studied in the literature, this seems to be the first time that data types for differential calculus have been proposed, which brings this subject into the discipline of domain theory and type theory.

We now mention briefly other approaches to differential calculus in domain theory, computable analysis, differential inclusions and interval analysis. In Martin (2000), using some domain-theoretic ideas, a notion of informatic derivative is defined, which for a $C^{1}$ function evaluates at each point to the absolute value of the derivative at that point. In computable analysis, the relation between the computability of a function and its derivative has been investigated (Pour-El and Richards 1988). In Weihrauch (2000), a representation of a $C^{1}$ function is given, by brute force, such that the representation of the function and that of its derivative, using a type 2 machine, are both computable. The relationship between the domain-theoretic derivative and the Clarke's derivative, mentioned above, gives a connection between domain theory and the area of analysis 
known as Differential Inclusions (Aubin and Cellina 1984) with applications in control theory. Finally, interval analysis (Moore 1966) provides an interval version of the Euler method for solving ordinary differential equations with lower and upper bounds at each stage of computation for the unique solution of an initial value problem given by a Lipschitz vector field. However, when implemented with floating point arithmetic, the interval containing the solution at a given point may get unduly large and convergence to the solution can no longer be guaranteed. Using the domain-theoretic Picard theorem, one obtains interval approximations to the solution, which when implemented with rational arithmetic, are still guaranteed to converge to the solution; for more details, see the follow-up papers Edalat et al. (2003) and Edalat and Pattinson (2003).

\section{The interval domain function spaces}

We assume the reader is familiar with the basic notions in domain theory; our main references for the subject are Gierz et al. (1980), Abramsky and Jung (1994), StoltenbergHansen et al. (1994), and Amadio and Curien (1998). For any dcpo (directed complete partial order) $A$ and $x \in A$, we write $\uparrow x=\{y \in A \mid x \sqsubseteq y\}$ and $\uparrow x=\{y \in A \mid x \ll y\}$. Let $\operatorname{Con}_{(A, \sqsubseteq)}$ and $\operatorname{Con}_{(A, \ll)}$ denote the consistency predicates with respect to $\sqsubseteq$ and $\ll$, respectively, that is,

$$
\begin{aligned}
& \operatorname{Con}_{(A, \sqsubseteq)}\left(a_{1}, a_{2}, \ldots a_{n}\right) \Longleftrightarrow \exists a \in A \cdot a_{i} \sqsubseteq a \text { for all } 1 \leqslant i \leqslant n . \\
& \operatorname{Con}_{(A, \ll)}\left(a_{1}, a_{2}, \ldots a_{n}\right) \Longleftrightarrow \exists a \in A \cdot a_{i} \ll a \text { for all } 1 \leqslant i \leqslant n .
\end{aligned}
$$

We often write $\operatorname{Con}_{\sqsubseteq}$ and $\operatorname{Con}_{\ll}$ for $\operatorname{Con}_{(A, \sqsubseteq)}$ and $\operatorname{Con}_{(A, \ll)}$, respectively. For dcpo's $\left(D, \sqsubseteq_{D}\right)$ and $\left(E, \sqsubseteq_{E}\right)$, the single-step function $a \searrow b: D \rightarrow E$ is defined by

$$
(a \searrow b)(x)= \begin{cases}b & \text { if } a \ll x \\ \perp & \text { otherwise. }\end{cases}
$$

In this paper, we will only work with continuous Scott domains (that is, bounded complete $\omega$-continuous dcpo's). The general form of a step function from a continuous Scott domain $\left(D, \sqsubseteq_{D}\right)$ to another one $\left(E, \sqsubseteq_{E}\right)$ is as follows. Consider the collection $\left(a_{i} \searrow b_{i}\right)_{i \in I}$ where $I$ is a finite set. Define the function space consistency predicate $\operatorname{Con}_{D \rightarrow E}(u)$ by

$$
\operatorname{Con}_{D \rightarrow E}\left(\left(a_{i} \searrow b_{i}\right)_{i \in I}\right) \Longleftrightarrow \forall J \subseteq I .\left(\operatorname{Con}_{(D, \ll)}\left(\left\{a_{i} \mid i \in J\right\}\right) \Rightarrow \operatorname{Con}_{(E, \sqsubseteq)}\left(\left\{b_{i} \mid i \in J\right\}\right)\right) .
$$

Then the lub $\bigsqcup_{i \in I} a_{i} \searrow b_{i}$ exists iff $\operatorname{Con}_{D \rightarrow E}\left(\left(a_{i} \searrow b_{i}\right)_{i \in I}\right)$. When the lub exists, it takes a finite number of values and is given by

$$
\left(\bigsqcup_{i \in I} a_{i} \searrow b_{i}\right)(x)=\bigsqcup_{a_{i} \ll x} b_{i}
$$

The way-below ordering of step functions is given by

$$
\left(\bigsqcup_{i \in I} a_{i} \searrow b_{i}\right) \ll\left(\bigsqcup_{j \in J} c_{j} \searrow d_{j}\right) \Longleftrightarrow \forall i \in I . b_{i} \ll \bigsqcup_{c_{j} \sqsubseteq a_{i}} d_{j} .
$$


For Scott continuous domains $D$ and $E$, the collection of all lubs $\left(\bigsqcup_{i \in I} a_{i} \searrow b_{i}\right)$ of consistent step functions with $a_{i} \in B$ and $b_{i} \in B^{\prime}$, where $B$ and $B^{\prime}$ are bases for $D$ and $E$, respectively, gives a basis for $D \rightarrow E$.

We consider the function spaces $D^{0}[0,1]=(\mathbf{I}[0,1] \rightarrow \mathbf{I R})$ and $D_{\mathrm{r}}^{0}[0,1]=([0,1] \rightarrow \mathbf{I} \mathbb{R})$; here $\mathbf{I}[0,1]$ is the continuous Scott domain of the compact intervals of $[0,1]$ ordered by reverse inclusion, and $\mathbf{I R}$ is the continuous Scott domain of the compact intervals of $\mathbb{R}$ together with $\mathbb{R}$, ordered by reverse inclusion. Note that $D^{0}[0,1]$ is the collection of Scott continuous interval-valued functions of an interval variable, whereas $D_{\mathrm{r}}^{0}[0,1]$ is the collection of Scott continuous interval-valued functions of a real variable; hence the subscript $r$ in $D_{\mathrm{r}}^{0}[0,1]$. We sometimes write $D^{0}$ for $D^{0}[0,1]$ and $D_{\mathrm{r}}^{0}$ for $D_{\mathrm{r}}^{0}[0,1]$. The choice of $[0,1]$ is simply for convenience. In practice, we sometimes replace $[0,1]$ by a non-trivial compact interval $a=[a, \bar{a}]$, a relatively compact open interval $a^{\circ}=(\underline{a}, \bar{a})$ (with $\underline{a}<\bar{a}$ ) or even $\mathbf{I} \mathbb{R}$. Recall that any continuous function $f:[0,1] \rightarrow \mathbb{R}$ has a canonical extension $\mathbf{I} f: \mathbf{I}[0,1] \rightarrow \mathbf{I} \mathbb{R}$ given by $(\mathbf{I} f)(x)=f(x)$, for $x \in \mathbf{I}[0,1]$, where $f(x)$ is the direct image of the interval $x$. In particular, the three basic arithmetic functions, addition, subtraction and multiplication, extend pointwise to Scott continuous functions $\mathbf{I R} \rightarrow \mathbf{I} \mathbb{R}$ which, for convenience, we denote simply by,+- and $\times$, that is, for $x, y \in \mathbf{I R}$ we write $x * y=\{u * v \mid u \in x, v \in y\}$ where $* \in\{+,-, \times\}$. As usual, we set $a * b=\perp$ if $a=\perp$ or $b=\perp$ for $* \in\{+,-, \times\}$.

A function $f:[0,1] \rightarrow \mathbb{I} \mathbb{R}$, given by two functions $f^{-}, f^{+}:[0,1] \rightarrow \mathbb{R}$ with $f(x)=$ $\left[f^{-}(x), f^{+}(x)\right]$, is continuous with respect to the Euclidean topology on $[0,1]$ and the Scott topology on IIR iff $f^{-}$and $f^{+}$are lower and upper semi-continuous functions, respectively. In this case, $f$ will have a Scott continuous extension to $\mathbf{I}[0,1]$, which, by overloading notation, we denote by $\mathbf{I} f=\mathbf{I}\left[f^{-}, f^{+}\right]: \mathbf{I}[0,1] \rightarrow \mathbf{I} \mathbb{R}$; it is given by $\mathbf{I} f(x)=\prod\{f(y) \mid y \in$ $x\}$. Conversely, the restriction of a Scott continuous function $f \in D^{0}$ to the maximal elements induces lower and upper semi-continuous functions $f^{-}, f^{+}:[0,1] \rightarrow \mathbb{R}$ given by $f(\{x\})=\left[f^{-}(x), f^{+}(x)\right]$. Clearly, $f$ preserves maximal elements iff $f^{-}=f^{+}$, in which case the induced map $f^{-}=f^{+}:[0,1] \rightarrow \mathbb{R}$ is continuous with respect to the Euclidean topology; for convenience, we denote this induced map simply by $f$ itself and write 'the induced map $f:[0,1] \rightarrow \mathbb{R}^{\prime}$.

The mapping $\mathscr{E}: D_{\mathrm{r}}^{0} \rightarrow D^{0}$ with $\mathscr{E}(f)=\mathbf{I} f$ is Scott continuous and injective. Thus we consider $D_{\mathrm{r}}^{0}$ as a subdomain of $D^{0}$. The Scott continuous map $\mathscr{I}: D^{0} \rightarrow D_{\mathrm{r}}^{0}$ with $\mathscr{I}: f \mapsto \lambda x . f(\{x\})$ in effect sends a function to its restriction on the maximal elements of $\mathbf{I}[0,1]$. We have $\mathscr{I} \circ \mathscr{E}=1_{D_{\mathrm{r}}^{0}}$ and $\mathscr{E} \circ \mathscr{I} \sqsupseteq 1_{D^{0}}$ where $1_{A}$ is the identity function on the dcpo $A$. In fact, we put $\mathscr{M}=\mathscr{E} \circ \mathscr{I}$; then $\mathscr{M}(f)$ is the greatest function in $D^{0}$ whose restriction to the maximal elements coincides with that of $f$ (Edalat and Escardo 2000). We can deduce the action of $\mathscr{I}$ and $\mathscr{E}$ on step functions. We write $a \searrow_{A} b$ for the single-step function $a \searrow b$ in the domain $A$. However, since we will only be concerned with step functions in $D^{0}$ and $D_{\mathrm{r}}^{0}$, we just write $a \searrow_{D^{0}} b$ simply as $a \searrow b$ and $a \searrow_{D_{\mathrm{r}}^{0}} b$ as $a \searrow_{\mathrm{r}} b$. For any finite set $I$ we have $\mathscr{I}\left(\bigsqcup_{i \in I} a_{i} \searrow b_{i}\right)=\bigsqcup_{i \in I} a_{i} \searrow_{\mathrm{r}} b_{i}$ while

$$
\mathscr{E}\left(\bigsqcup_{i \in I} a_{i} \searrow_{\mathrm{r}} b_{i}\right)=\bigsqcup\left\{\left(\sqcap_{j \in J} a_{j}\right) \searrow \sqcap_{j \in J} b_{j} \mid J \subseteq I \text { with } \bigcup_{j \in J} a_{j}^{\circ} \text { connected }\right\}
$$


These formulas extend by continuity to all suprema of step functions. We have

$$
\operatorname{Con}_{D^{0}}\left(a_{i} \searrow b_{i}\right)_{i \in I} \Longleftrightarrow \operatorname{Con}_{D_{\mathrm{r}}^{0}}\left(a_{i} \searrow_{\mathrm{r}} b_{i}\right)_{i \in I} \text {. }
$$

The step functions $\left(a_{i} \searrow b_{i}\right)_{i \in I} \in D^{0}$ and $\left(a_{i} \searrow_{\mathrm{r}} b_{i}\right)_{i \in I} \in D_{\mathrm{r}}^{0}$ are called rational step functions of $D^{0}$ and $D_{\mathrm{r}}^{0}$, respectively, if $a_{i}$ and $b_{i}$ are rational intervals for all $i \in I$. The collection of rational step functions of $D^{0}$ (respectively, $D_{\mathrm{r}}^{0}$ ) gives a countable basis for $D^{0}$ (respectively, $D_{\mathrm{r}}^{0}$ ). It is convenient to define a more general basis for $D^{0}$ and $D_{\mathrm{r}}^{0}$ as follows. We refer to a polynomial with rational coefficients as a rational polynomial.

Definition 2.1. A continuous function $p:[v, w] \rightarrow \mathbb{R}$ is a piecewise (rational) polynomial if there exists a partition $v=c_{0}<c_{1} \cdots<c_{n-1}<c_{n}=w$ of $[v, w]$ such that $c_{i}(i=0, \cdots, n)$ are real (rational) numbers and each restriction $p \uparrow_{\left[c_{i-1}, c_{i}\right]}$ is a (rational) polynomial. We call $p$ a piecewise semi-rational polynomial if $c_{i}(i=0, \cdots, n)$ are algebraic numbers and each restriction $p \uparrow_{\left[c_{i-1}, c_{i}\right]}$ is a rational polynomial up to an additive algebraic number (that is, all the coefficients are rational, except possibly the constant term which is an algebraic number).

Let $a \in \mathbf{I}[0,1]$ and let $p, q:[\underline{a}, \bar{a}] \rightarrow \mathbb{R}$ be piecewise (rational or semi-rational) polynomials satisfying $p(x) \leqslant q(x)$ for $x \in a^{\circ}$. We define the (rational or semi-rational) polynomial step function $a \searrow[p, q]: \mathbf{I}[0,1] \rightarrow \mathbf{I} \mathbb{R}$ by

$$
(a \searrow[p, q])(x)= \begin{cases}\mathbf{I}[p, q](x) & \text { if } a \ll x \\ \perp & \text { otherwise. }\end{cases}
$$

Clearly, by choosing $p$ and $q$ to be the constant polynomials $\underline{b}$ and $\bar{b}$, we obtain an ordinary rational step function, that is, $a \searrow[\underline{b}, \bar{b}]=a \searrow b$. The consistency relation for a finite set of polynomial step functions is given by $\left\{a_{i} \searrow\left[p_{i}, q_{i}\right] \mid i \in I\right\} \in \mathrm{Con}_{0}$ iff

$$
\forall J \subseteq I . \operatorname{Con}_{\ll}\left\{a_{i} \mid i \in J\right\} \Rightarrow \forall i, k \in J \forall x \in\left(\bigsqcup_{j \in J} a_{j}\right)^{\circ} \cdot p_{i}(x) \leqslant q_{k}(x) .
$$

The collection of consistent finite sets of rational (or semi-rational) polynomial step functions forms a basis for $D^{0}$, which we call the rational (or semi-rational) polynomial basis. We also get a similar basis for $D_{\mathrm{r}}^{0}$ with its similar consistency relation; in fact $\left(a \searrow_{\mathrm{r}}[p, q]\right)=\mathscr{I}(a \searrow[p, q])$ is given by

$$
\left(a \searrow_{r}[p, q]\right)(x)= \begin{cases}\mathbf{I}[p, q](x) & \text { if } x \in a^{\circ} \\ \perp & \text { otherwise. }\end{cases}
$$

Consider a step function $f=\bigsqcup_{i \in I}\left\{\left(a_{i} \searrow b_{i}\right)\right\}$. The end points of the compact intervals $a_{i},(i \in I)$, partition $[0,1]$ into a finite number of intervals (open, closed or half-open, halfclosed) of $[0,1]$, called the associated intervals of the step function, with the property that $f(\{x\})$ is a constant compact interval for $x$ in each of these intervals. For a polynomial step function $f=\bigsqcup_{i \in I}\left\{\left(a_{i} \searrow\left[p_{i}, q_{i}\right]\right)\right\}$, the compact interval $f(\{x\})$ is bounded by two fixed piecewise polynomials in each of the associated intervals.

We define the domain of $h: \mathbf{I}[0,1] \rightarrow \mathbf{I R}$ by

$$
\operatorname{Dom}(h)=\{x \in[0,1] \mid h(\{x\}) \neq \perp\} .
$$




\subsection{Embedding of classical functions}

Let $C^{0}[0,1]$ be, as usual, the set of real-valued continuous functions on $[0,1]$ with the sup norm; its topology is the same as the compact open topology. Furthermore, for an integer $k \geqslant 1$, we let $C^{k}[0,1]$ denote the set of functions with continuous $k$ th derivative on $[0,1]$ equipped with the norm $\|f\|=\max \left\{\sup \left|f^{(n)}\right| \mid 0 \leqslant n \leqslant k\right\}$, where $f^{(n)}$ is the $n$th derivative of $f$.

Define $\Gamma^{0}: C^{0}[0,1] \rightarrow D^{0}[0,1]$ by $\Gamma^{0}(f)=\mathbf{I} f$. Recall that an embedding is a continuous injection that maps open subsets to relatively open subsets in its image.

Theorem 2.2. The mapping $\Gamma^{0}$ is an embedding into a proper subset of the maximal elements of $D^{0}$

Proof. We use the way-below relation both for domains such as $D^{0}$ and for the lattice $\Omega X$ of the open subsets of a locally quasi-compact topological space $X$.

The function $g: \mathbf{I}[0,1] \rightarrow \mathbf{I} \mathbb{R}$ with

$$
g(x)= \begin{cases}\{0\} & \text { if } \bar{x}<1 / 2 \\ \{1\} & \text { if } \underline{x}>1 / 2 \\ {[0,1]} & \text { if } 1 / 2 \in x\end{cases}
$$

is maximal but is not the extension of any function in $C^{0}[0,1]$.

Continuity: We have

$$
\mathbf{I} f=\bigsqcup_{i \in I}\left\{a_{i} \searrow b_{i} \mid a_{i} \searrow b_{i} \ll D^{0} \mathbf{I} f\right\}=\bigsqcup_{i \in I}\left\{a_{i} \searrow b_{i} \mid \uparrow a_{i} \ll \Omega \mathbf{I}[0,1](\mathbf{I} f)^{-1}\left(\uparrow b_{i}\right)\right\} .
$$

Hence any open set containing $\mathbf{I} f$ contains a step function $\bigsqcup_{i \in J} a_{i} \searrow b_{i}$, for some finite indexing set $J$, with $\uparrow a_{i} \ll f^{-1}\left(\uparrow b_{i}\right)(i \in J)$ that contains $\mathbf{I} f$. But the open set $\bigcap_{i \in J}\left(a_{i}, b_{i}^{\circ}\right)$ (of the compact open topology) contains $f$ and is mapped into $\uparrow\left(\bigsqcup_{i \in J} a_{i} \searrow b_{i}\right)$.

Openness: Let $(C, O)$ be an open set of the compact-open topology containing the continuous $f:[0,1] \rightarrow \mathbb{R}$. Then there exists a decreasing sequence $C_{i}$ of finite unions of intervals such that $C=\bigcap_{i \in \omega} C_{i}$. By Scott continuity of $\mathbf{I} f$, there exists an $i \in \omega$ such that $(\mathbf{I} f)\left(C_{i}\right)=f\left(C_{i}\right) \subseteq O$. Suppose $C_{i}=\bigcup_{j=1}^{n} A_{j}$ where each $A_{j}$ is a compact interval. Since $f\left(A_{j}\right)$ is compact, there exist open sets $B_{j}$ with $f\left(A_{j}\right) \subseteq B_{j} \subseteq O$ and $\overline{B_{j}} \subseteq O$, for $1 \leqslant j \leqslant n$. Then $\mathbf{I} f \in \hat{\uparrow}\left(A_{j} \searrow B_{j}\right)$ for $1 \leqslant j \leqslant n$. Moreover, $\left(\Gamma^{0}\right)^{-1}\left(\uparrow\left(A_{j} \searrow B_{j}\right)\right) \subseteq(C, O)$. Hence $\Gamma^{0}$ is a topological embedding.

Recall that an adjunction between posets $A$ and $B$ is a pair of monotone maps $l: A \rightarrow B$, called a lower adjoint, and $u: B \rightarrow A$, called an upper adjoint, with $u \circ l \sqsupseteq 1_{A}$ and $l \circ u \sqsubseteq 1_{B}$. Since $\mathscr{I} \circ \mathscr{E}=1_{D_{\mathrm{r}}^{0}}$ and $\mathscr{E} \circ \mathscr{I} \sqsupseteq 1_{D^{0}}$, it follows that $\mathscr{I}$ is a lower adjoint. Thus, by Abramsky and Jung (1994, Proposition 3.1.14), $\mathscr{I}$ preserves the way-below relation and is therefore an open map, that is, one that preserves open subsets. Since $\mathscr{I}$ induces a bijection between the sets of maximal elements of $D^{0}$ and $D_{\mathrm{r}}^{0}$, it follows that the map $\Gamma_{\mathrm{r}}^{0}=\mathscr{I} \circ \Gamma^{0}: C^{0} \rightarrow D_{\mathrm{r}}^{0}$ sends any open subset to a relatively open subset in its image and we obtain the following corollary.

Corollary 2.3. $\Gamma_{\mathrm{r}}^{0}: C^{0} \rightarrow D_{\mathrm{r}}^{0}$ is an embedding into a proper subset of the maximal elements of $D_{\mathrm{r}}^{0}$. 


\section{Primitive maps of a Scott continuous function}

In classical analysis, the primitive maps, or the indefinite integrals, of a continuous function $g:[0,1] \rightarrow \mathbb{R}$ can be considered as the family of continuous functions $\{f+a \mid a \in \mathbb{R}\}$ where $f:[0,1] \rightarrow \mathbb{R}$ is differentiable with $f^{\prime}=g$. In this section, we seek the analogous concept in domain theory. In order to define the primitive maps of a Scott continuous function, which in the spirit of classical analysis is expected to be a family of Scott continuous functions, we first introduce the notion of an interval Lipschitz constant of a Scott continuous function. Note that, in this paper, we write the classical derivative of a classical function $f$ as $f^{\prime}$. The notation $\frac{d f}{d x}$ is used only for the domain-theoretic derivative to be introduced in the next section. The type of a variable is always clear from the context, which the reader should identify.

\subsection{Interval Lipschitz constant}

The idea of an interval Lipschitz constant can be motivated by considering the case of a classical function or its canonical interval domain extension. Suppose that, for some open interval $a \subseteq[0,1]$ and a compact interval $b \subseteq \mathbb{R}$, the function $f:[0,1] \rightarrow \mathbb{R}$ satisfies $\underline{b}(x-y) \leqslant f(x)-f(y) \leqslant \bar{b}(x-y)$ for all $x, y \in a^{\circ}$ with $y \leqslant x$; note that if $f$ is in fact $C^{1}$, this will be equivalent to $\underline{b} \leqslant f^{\prime}(x) \leqslant \bar{b}$ for all $x \in a^{\circ}$. We say that $\underline{b}$ and $\bar{b}$ are, respectively, a lower Lipschitz constant and an upper Lipschitz constant for $f$ in the open interval $a$. Then we can think of $b$ as an interval Lipschitz constant for $f$ in $a$. The above inequalities can be expressed in terms of $\mathbf{I} f: \mathbf{I}[0,1] \rightarrow \mathbf{I} \mathbb{R}$ to give: $b(\{x\}-\{y\}) \sqsubseteq \mathbf{I} f(\{x\})-\mathbf{I} f(\{y\})$ for all $\{x\},\{y\} \gg a$. This latter condition is now generalised as follows to define the notion of an interval Lipschitz constant.

Definition 3.1. The continuous function $f: \mathbf{I}[0,1] \rightarrow \mathbf{I} \mathbb{R}$ has an interval Lipschitz constant $b \in \mathbf{I} \mathbb{R}$ in $a \in \mathbf{I}[0,1]$ if $f$ satisfies the following differential property: for all $x_{1}, x_{2} \in \mathbf{I}[0,1]$ with $a \ll x_{1}$ and $a \ll x_{2}, b\left(x_{1}-x_{2}\right) \sqsubseteq f\left(x_{1}\right)-f\left(x_{2}\right)$. The single-step tie $\delta(a, b) \subseteq D^{0}[0,1]$ of $a$ with $b$ is the collection of all functions in $D^{0}[0,1]$ that have an interval Lipschitz constant $b$ in $a$.

\section{Proposition 3.2.}

(i) If $f \in \delta(a, b)$, for $a^{\circ} \neq \varnothing$ and $b \neq \perp$, then $f(\{x\})$ is maximal for each $x \in a^{\circ}$ and the induced function $f:(\underline{a}, \bar{a}) \rightarrow \mathbb{R}$ is Lipschitz and for all $u, v \in a^{\circ}$ with $v \leqslant u$ we have $\underline{b}(u-v) \leqslant f(u)-f(v) \leqslant \bar{b}(u-v)$.

(ii) For $f:(\underline{a}, \bar{a}) \rightarrow \mathbb{R}$, with $a \subseteq[0,1]$ a non-trivial interval, we have $\mathbf{I} f \in \delta(a, b)$ iff $f$ is Lipschitz and for all $u, v \in a^{\circ}$ with $v \leqslant u$ we have $\underline{b}(u-v) \leqslant f(u)-f(v) \leqslant \bar{b}(u-v)$.

Proof.

(i) Suppose $f \in \delta(a, b)$, but $f(\{y\})$ is not maximal. Let $\epsilon>0$ be small enough that $x=[y-\epsilon, y+\epsilon] \subseteq a^{\circ}$. The diameter of $b(x-x)$ can be made arbitrarily small by choosing $\epsilon$ small. However, since $b(x-x) \sqsubseteq f(x)-f(x)$, it follows that the diameter of $b(x-x)$ is larger than the diameter of $f(x)-f(x)$, which is larger than the diameter of $f(\{y\})$. This contradiction establishes the proof.

(ii) If $\mathbf{I} f \in \delta(a, b)$ and $\underline{a}<v \leqslant u<\bar{a}$, then, by putting $x=\{u\}$ and $y=\{v\}$, we get $b(\{u\}-\{v\}) \sqsubseteq \mathbf{I} f(\{u\}-\mathbf{I} f(\{v\})$, in other words $\underline{b}(u-v) \leqslant f(u)-f(v) \leqslant \bar{b}(u-v)$. For 


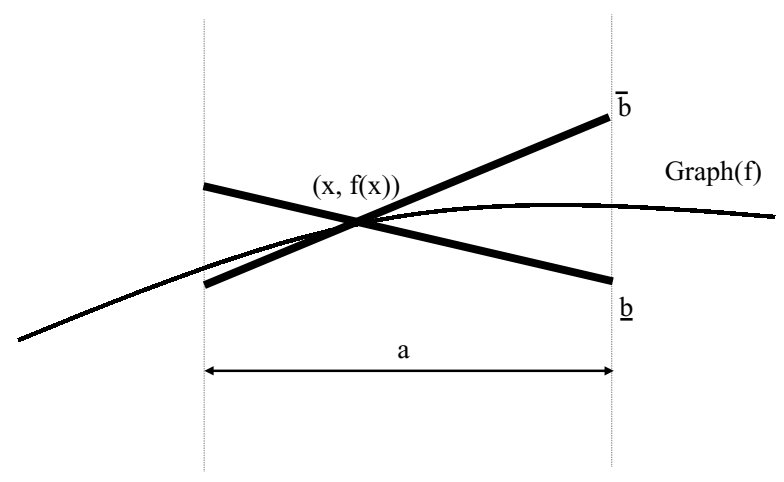

Fig. 1. Geometric interpretation of interval Lipschitz constant

the other implication, assume that $x, y \gg a$ and $z \in \mathbf{I} f(x)-\mathbf{I} f(y)$. Then $z=f(u)-f(v)$ for some $u \in x$ and $v \in y$. Hence, $\frac{z}{u-v} \in b$ and it follows that $z \in b(x-y)$.

From Proposition 3.2, we get a simple geometric characterisation for $\mathbf{I} f \in \delta(a, b)$ for a classical function $f \in C^{0}[0,1]$. Let $L(x, \underline{b})$ and $L(x, \bar{b})$ be the straight lines through the point $(x, f(x))$, for $x \in a^{\circ}$, with slopes $\underline{b}$ and $\bar{b}$ respectively. We have $\mathbf{I} f \in \delta(a, b)$ iff the graph of $f$ at $(x, f(x))$, for each $x \in a^{\circ}$, is locally contained within the closed region bounded anti-clockwise from $L(x, \underline{b})$ to $L(x, \bar{b})$ (see Figure 1$)$.

\section{Example 3.3.}

(i) If $f_{1}: x \mapsto|x|: \mathbb{R} \rightarrow \mathbb{R}$ is the absolute value function and $a \subset \mathbb{R}$ is any compact interval with $0 \in a^{\circ}$, then $\mathbf{I} f_{1} \in \delta(a,[-1,1])$.

(ii) If $f_{2}: x \mapsto x^{2} \sin \frac{1}{x}: \mathbb{R} \rightarrow \mathbb{R}$ (with $f(0)=0$ ), then $\mathbf{I} f_{2} \in \delta([-1,1],[-3,3]$ ).

(iii) If $f_{3}: x \mapsto x \sin \frac{1}{x}: \mathbb{R} \rightarrow \mathbb{R}$ (with $f(0)=0$ ), then, for any compact interval $a$ with $0 \in a^{\circ}$, we have $\mathbf{I} f_{2} \in \delta(a, b)$ iff $b=\perp$.

(iv) If $g \in D^{0}[0,1]$ is the function given by Equation 2, then, for any compact interval $a \subseteq[0,1]$ with $1 / 2 \in a^{\circ}$, we have $g \in \delta(a, b)$ iff $b=\perp$.

The following proposition justifies our definition of interval Lipschitz constant.

Proposition 3.4. For $f \in C^{1}[0,1]$, the following conditions are equivalent:

(i) $\mathbf{I} f \in \delta(a, b)$.

(ii) $\forall z \in a^{\circ} . \underline{b} \leqslant f^{\prime}(z) \leqslant \bar{b}$.

(iii) $a \searrow b \sqsubseteq \mathbf{I} f^{\prime}$.

Proof.

(i) $\Longleftrightarrow$ (ii). This follows from Proposition 3.2 and the mean value theorem.

(iii) $\Rightarrow$ (ii). Since $a \ll\{z\}$ for all $z \in a^{\circ}$, it follows from $a \searrow b \sqsubseteq \mathbf{I} f^{\prime}$ that $b \sqsubseteq \mathbf{I} f^{\prime}\{z\}$ for $a \ll\{z\}$.

(ii) $\Rightarrow$ (iii). From (ii), we get $b \sqsubseteq \mathbf{I} f^{\prime}(x)$ for $a \ll x$. Hence $a \searrow b \sqsubseteq \mathbf{I} f^{\prime}$.

For each rational $a$ and rational $b$, the tie $\delta(a, b)$ is a family of functions in $D^{0}$ with a finitary differential property as specified in Definition 3.1; similarly, $\operatorname{Im}\left(\Gamma^{0}\right) \cap \delta(a, b)$ gives 
a family of functions in $C^{0}$ with a finitary differential property. Note that:

(i) $f \sqsubseteq g \Rightarrow(f \in \delta(a, b) \Rightarrow g \in \delta(a, b))$, that is, $\delta(a, b)$ is an upper subset of $\mathbf{I}[0,1] \rightarrow \mathbf{I} \mathbb{R}$.

(ii) If $f=g+\{c\}$ for some $c \in \mathbb{R}$, then $f \in \delta(a, b) \Longleftrightarrow g \in \delta(a, b)$.

We will see at the end of this section that $\delta(a, b)$ is defined as the set of primitive maps of $a \searrow b$. However, in order to define the set of the primitive maps of a Scott continuous function in general, we first need to study and generalise the notion of single-step ties.

\subsection{Ties and their properties}

We will now study the single-step ties and their generalisations. First we note that single-step ties share some common properties with single-step functions.

Proposition 3.5. Suppose $a^{\circ} \neq \varnothing$ and $b \neq \perp$. We have $\delta(a, b) \supseteq \delta(c, d)$ iff $c \sqsubseteq a$ and $b \sqsubseteq d$.

Proof. The 'If' part follows immediately from the definition of $\delta(a, b)$. To show the 'Only if' part, assume $\delta(a, b) \supseteq \delta(c, d)$ holds but not $c \sqsubseteq a$. Let $g:[0,1] \rightarrow \mathbb{R}$ be the linear partial function defined by

$$
g(x)= \begin{cases}\underline{d x} & x \in c^{\circ} \\ (\underline{b}-1) x & x \in(a \backslash c)^{\circ} \\ \perp & \text { otherwise. }\end{cases}
$$

Then $\mathbf{I} g \in \delta(c, d)$ but $\mathbf{I} g \notin \delta(a, b)$. On the other hand, if $b \sqsubseteq d$ does not hold, consider the function $f: c^{\circ} \rightarrow \mathbb{R}$ with $f(x)=t x$ for $t \in d \backslash b$. Then, $\mathbf{I} f \in \delta(c, d) \backslash \delta(a, b)$.

Corollary 3.6. Suppose $a^{\circ}, c^{\circ} \neq \varnothing$ and $b, d \neq \perp$. We have $\delta(a, b) \supseteq \delta(c, d)$ iff $a \searrow b \sqsubseteq c \searrow d$. Corollary 3.7. Suppose $a^{\circ}, c^{\circ} \neq \varnothing$ and $b, d \neq \perp$. We have $\delta(a, b)=\delta(c, d) \Longleftrightarrow a=c \& b=d$.

By analogy with step functions, we now have the following definition.

Definition 3.8. A step tie of $D^{0}$ is any non-empty finite intersection $\bigcap_{1 \leqslant i \leqslant n} \delta\left(a_{i}, b_{i}\right) \subset D^{0}$. A tie of $D^{0}$ is any non-empty intersection $\Delta=\bigcap_{i \in I} \delta\left(a_{i}, b_{i}\right) \subset D^{0}$. The domain of $\Delta$ is $\operatorname{dom}(\Delta)=\bigcup_{i \in I}\left\{a_{i}^{\circ} \mid b_{i} \neq \perp\right\}$.

A step tie with rational intervals gives us a family of functions with a finite set of given differential properties, and a tie gives a family of functions with a set of given differential properties. In a similar way to Proposition 3.2, we have the following result. Recall that a function $f: O \rightarrow \mathbb{R}$ defined on the open set $O \subseteq \mathbb{R}$ is locally Lipschitz if it is Lipschitz in a neighbourhood of any point in $O$.

Proposition 3.9. If $\Delta \subset D^{0}$ is a tie and $f \in \Delta$, then $f(\{x\})$ is maximal for $x \in \operatorname{dom}(\Delta)$ and the induced real-valued function on $\operatorname{dom}(\Delta)$ is locally Lipschitz. In particular, for $f \in C^{0}$, if $\mathbf{I} f \in \Delta$, then $f$ is locally Lipschitz in $\operatorname{dom}(\Delta)$.

Proof. Let $x \in \operatorname{dom}(\Delta)$. Then there exists a step tie $\delta(a, b)$ with $x \in a^{\circ}$ and $\Delta \subseteq \delta(a, b)$ and the result follows from Proposition 3.2.

We now aim to establish a necessary and sufficient condition for a family of step ties to be consistent. Let $g \in D^{0}$ with $g=\bigsqcup_{i \in I} a_{i} \searrow b_{i}$ and put $g(\{x\})=\left[g^{-}(x), g^{+}(x)\right]$. Assume 
$k: \operatorname{dom}(g) \rightarrow \mathbb{R}$ is any Lebesgue integrable function with $g^{-} \leqslant k \leqslant g^{+}$and let $\mu$ be the Lebesgue measure on $[0,1]$. Let $\left(O_{t}\right)_{t \in C}$ be the set of connected components of $\operatorname{dom}(\mathrm{g})$ and fix $c_{t} \in O_{t}$ for each $t \in C$. Define

$$
\begin{aligned}
f:[0,1] & \rightarrow \mathbb{R}_{\perp} \\
x & \mapsto \begin{cases}\int_{c_{t}}^{x} k d \mu & x \in O_{t} \\
\perp & x \notin \operatorname{dom}(g)\end{cases}
\end{aligned}
$$

Lemma 3.10. With $f$ as defined above, we have $\mathbf{I} f \in \bigcap_{i \in I} \delta\left(a_{i}, b_{i}\right)$.

Proof. We show that $\mathbf{I} f \in \delta\left(a_{i}, b_{i}\right)$ for each $i \in I$. Let $x, y \in a_{i}^{\circ}$ with $x>y$. For any $z \in a_{i}^{\circ}$, we have $b_{i} \sqsubseteq g\{z\}$. Hence

$$
\underline{b_{i}} \leqslant g^{-}(z) \leqslant k(z) \leqslant g^{+}(z) \leqslant \overline{b_{i}},
$$

which implies that $[x, y] \subseteq \operatorname{dom}(g)$, that is, $x$ and $y$ belong to the same component $O_{t}$, say, of $\operatorname{dom}(g)$. This gives $f(x)-f(y)=\int_{c_{t}}^{x} k d \mu-\int_{c_{t}}^{y} k d \mu=\int_{y}^{x} k d \mu$. By Equation 3 , we obtain $\underline{b_{i}}(x-y) \leqslant f(x)-f(y) \leqslant \overline{b_{i}}(x-y)$, that is, $\mathbf{I} f \in \delta\left(a_{i}, b_{i}\right)$.

Proposition 3.11. For any indexing set $I, \bigcap_{i \in I} \delta\left(a_{i}, b_{i}\right) \neq \varnothing$ iff the family of step functions $\left(a_{i} \searrow b_{i}\right)_{i \in I}$ is consistent.

Proof. In order to show the 'Only if' part, suppose there exists $f \in \bigcap_{i \in I} \delta\left(a_{i}, b_{i}\right)$ but $\left(a_{i} \searrow b_{i}\right)_{i \in I}$ is not consistent. Then there is a finite subfamily $\left(a_{i} \searrow b_{i}\right)_{i \in J}$, for $J \subseteq I$, which is not consistent, that is, $\operatorname{Con}_{\ll}\left(a_{i}\right)_{i \in J}$ but not $\operatorname{Con}_{\sqsubseteq}\left(b_{i}\right)_{i \in J}$. Therefore there exists a pair in this finite subfamily (say $\delta\left(a_{1}, b_{1}\right)$ and $\delta\left(a_{2}, b_{2}\right)$ ) such that $a_{1}^{\circ} \cap a_{2}^{\circ} \neq \varnothing$ with $b_{1} \cap b_{2}=\varnothing$. Assume without loss of generality that $\overline{b_{2}}<\underline{b_{1}}$. Take maximal elements $\left\{x_{1}\right\}$ and $\left\{x_{2}\right\}$ with $x_{2}<x_{1}$ and $x_{1}, x_{2} \in\left(a_{1} \cap a_{2}\right)^{\circ}$. Then $b_{1}\left(\left\{x_{1}\right\}-\left\{x_{2}\right\}\right)$ and $b_{2}\left(\left\{x_{1}\right\}-\left\{x_{2}\right\}\right)$ are disjoint.

Since, by assumption, $f \in \delta\left(a_{1}, b_{1}\right) \cap \delta\left(a_{2}, b_{2}\right)$, we have

$$
\begin{gathered}
b_{1}\left(\left\{x_{1}\right\}-\left\{x_{2}\right\}\right) \sqsubseteq f\left(\left\{x_{1}\right\}\right)-f\left(\left\{x_{2}\right\}\right) \\
b_{2}\left(\left\{x_{1}\right\}-\left\{x_{2}\right\}\right) \sqsubseteq f\left(\left\{x_{1}\right\}\right)-f\left(\left\{x_{2}\right\}\right),
\end{gathered}
$$

which gives a contradiction, since the two intervals on the left-hand side are disjoint.

For the 'If' part, by assumption, $g=\bigsqcup_{i \in I} a_{i} \searrow b_{i}$ exists and, thus, the result follows from Lemma 3.10 by taking $k=g^{-}$, say.

Corollary 3.12. $\bigcap_{i \in I} \delta\left(a_{i}, b_{i}\right) \neq \varnothing$ iff for any finite subfamily $J \subseteq I$ we have

$$
\bigcap_{i \in J} \delta\left(a_{i}, b_{i}\right) \neq \varnothing
$$

Proposition 3.13. $\delta(a, b) \supseteq \bigcap_{i \in I} \delta\left(a_{i}, b_{i}\right)$ if $a \searrow b \sqsubseteq \bigsqcup_{i \in I} a_{i} \searrow b_{i}$.

Proof. Let $a^{\prime} \searrow b^{\prime} \ll a \searrow b$ so that $b^{\prime} \ll \bigcup_{a_{i} \sqsubseteq a^{\prime}} b_{i}$. Assume $f \in \bigcap_{i \in I} \delta\left(a_{i}, b_{i}\right)$. We will show that $f \in \delta\left(a^{\prime}, b^{\prime}\right)$. Let $a^{\prime} \ll x_{1}, x_{2}$. For each $i \in I$ with $a_{i} \sqsubseteq a^{\prime}$ we have:

$$
b_{i}\left(x_{1}-x_{2}\right) \sqsubseteq f\left(x_{1}\right)-f\left(x_{2}\right) .
$$


By the Scott continuity of product of two intervals and our assumption, we get

$$
b^{\prime}\left(x_{1}-x_{2}\right) \sqsubseteq \bigcup_{a_{i} \sqsubseteq a} b_{i}\left(x_{1}-x_{2}\right) \sqsubseteq f\left(x_{1}\right)-f\left(x_{2}\right),
$$

which show that $f \in \delta\left(a^{\prime}, b^{\prime}\right)$. Now let $\left(a_{n}^{\prime} \searrow b_{n}^{\prime}\right)_{n \geqslant 0}$ be an increasing chain of single-step functions way-below $a \searrow b$ with lub $a \searrow b$. Then, we have $f \in \bigcap_{n \geqslant 0} \delta\left(a_{n}^{\prime}, b_{n}^{\prime}\right)$. Let $a \ll x_{1}, x_{2}$. Then we can find $n$ such that $a \ll a_{n} \ll x_{1}, x_{2}$ and thus

$$
b_{n}\left(x_{1}-x_{2}\right) \sqsubseteq f\left(x_{1}\right)-f\left(x_{2}\right) .
$$

By Scott continuity, we get

$$
b\left(x_{1}-x_{2}\right) \sqsubseteq f\left(x_{1}\right)-f\left(x_{2}\right),
$$

as required.

Corollary 3.14. $\bigcap_{i \in I} \delta\left(a_{i}, b_{i}\right) \supseteq \bigcap_{i \in J} \delta\left(a_{i}, b_{i}\right)$ if $\bigsqcup_{i \in I} a_{i} \searrow b_{i} \sqsubseteq \bigsqcup_{i \in J} a_{i} \searrow b_{i}$.

Let $\left(T^{1}[0,1], \supseteq\right)$ be the poset of ties of $D^{0}$ ordered by reverse inclusion.

Proposition 3.15. $\left(T^{1}[0,1]\right.$, 卫) is a dcpo.

Proof. Suppose $\left(\Delta_{j}\right)_{j \in J}$ is a directed set with respect to the partial order $\supseteq$, that is, $\Delta_{j_{1}} \cap \Delta_{j_{2}} \neq \varnothing$ for $j_{1}, j_{2} \in J$. Let $\Delta_{j}=\bigcap_{i \in I_{j}} \delta\left(a_{i}, b_{i}\right)$, where we assume $I_{j_{1}} \cap I_{j_{2}}=\varnothing$ for $j_{1} \neq j_{2}$. Consider the collection $\left(\delta\left(a_{i}, b_{i}\right)\right)_{i \in \cup_{j \in J} I_{j}}$. By Corollary 3.12, it suffices to show that any finite subfamily of this collection has non-empty intersection. Suppose $i_{t} \in \bigcup_{j \in J} I_{j}$ for $1 \leqslant t \leqslant n$. Then $\delta\left(a_{i_{t}}, b_{i_{t}}\right) \in \Delta_{j_{t}}$ for some $j_{t} \in I(1 \leqslant t \leqslant n)$. By assumption, $\bigcap_{1 \leqslant t \leqslant n} \Delta_{j_{t}} \neq \varnothing$. Hence, $\bigcap_{1 \leqslant t \leqslant n} \delta\left(a_{i_{t}}, b_{i_{t}}\right) \supseteq \bigcap_{1 \leqslant t \leqslant n} \Delta_{j_{t}} \neq \varnothing$.

We are finally in a position to define the set of primitive maps of a Scott continuous function; in fact we can now do more and define the integral operator that gives the set of primitive maps of any Scott continuous function as follows.

Definition 3.16. The integral operator $\int: D^{0} \rightarrow T^{1}$ is defined by $\int\left(\bigsqcup_{i \in I} a_{i} \searrow b_{i}\right)=\bigcap_{i \in I}$ $\delta\left(a_{i}, b_{i}\right)$. We usually write $\int(f)$ as $\int f$ and call it the set of primitive maps of $f$.

Proposition 3.17. The integral operator is well-defined, onto and continuous.

Proof. By Corollary 3.14, $\int$ is well-defined and monotone. By Proposition 3.11, it is onto. Let $\left(g_{i}\right)_{i \in I}$ be a directed set in $D^{0}$ with $g_{i}=\bigsqcup_{j \in I_{i}} a_{j} \searrow b_{j}$. Then,

$$
\begin{aligned}
\int \bigsqcup_{i \in I} g_{I} & =\int \bigsqcup_{i \in I} \bigsqcup_{j \in I_{i}} a_{j} \searrow b_{j} \\
& =\bigcap_{i \in I} a_{j \in I_{i}} \searrow b_{j} \\
& =\bigcap_{i \in I} \int g_{i} \\
& =\bigsqcup_{i \in I} \int g_{i} .
\end{aligned}
$$




\section{Derivative of a Scott continuous function}

Given a Scott continuous function $f: \mathbf{I}[0,1] \rightarrow \mathbf{I} \mathbb{R}$, the relation $f \in \delta(a, b)$, for some intervals $a$ and $b$, provides, as we have seen, finitary information about the local differential properties of $f$. By collecting all such local information, we obtain the complete differential properties of $f$, namely its derivative. For clarity, we will use $\frac{d f}{d x}$ to denote the new notion of the domain-theoretic derivative of a Scott continuous function $f$ and write $f^{\prime}$ for the classical derivative of a classical continuous function $f$.

Definition 4.1. The derivative of a continuous function $f: \mathbf{I}[0,1] \rightarrow \mathbf{I} \mathbb{R}$ is the map

$$
\frac{d f}{d x}=\bigsqcup_{f \in \delta(a, b)} a \searrow b: \mathbf{I}[0,1] \rightarrow \mathbf{I} \mathbb{R} .
$$

\section{Theorem 4.2.}

(i) $\frac{d f}{d x}$ is well-defined and Scott continuous.

(ii) If $f \in C^{1}[0,1]$, then $\frac{d \mathbf{I} f}{d x}=\mathbf{I} f^{\prime}$.

(iii) $f \in \delta(a, b)$ iff $a \searrow b \sqsubseteq \frac{d f}{d x}$.

\section{Proof.}

(i) Let the indexing set $I$ be defined by $i \in I \Longleftrightarrow f \in \delta\left(a_{i}, b_{i}\right)$. Then $\bigcap_{i \in I} \delta\left(a_{i}, b_{i}\right) \neq \varnothing$. Hence, $\left(a_{i} \searrow b_{i}\right)_{i \in I}$ is consistent. Therefore, $\frac{d f}{d x}=\bigsqcup_{i \in I} a_{i} \searrow b_{i}$ exists and is Scott continuous.

(ii) By Proposition 3.4, we have $\mathbf{I} f \in \delta(a, b) \Longleftrightarrow a \searrow b \sqsubseteq \mathbf{I} f^{\prime}$. Hence, $\mathbf{I} f^{\prime}=\bigsqcup_{\mathbf{I} f \in \delta(a, b)} a \searrow b$.

(iii) The left to right implication is obvious. For the right to left implication, we show that $a^{\prime} \searrow b^{\prime} \ll \frac{d f}{d x}$ implies $f \in \delta\left(a^{\prime}, b^{\prime}\right)$, from which the result follows. Assume that $a^{\prime} \searrow b^{\prime} \ll \frac{d f}{d x}$. Since $\frac{d f}{d x}$ is the directed lub of step functions whose corresponding simple ties contain $f$, there exists a step function $\left(a_{i} \searrow b_{i}\right)_{i \in I}$ with $f \in \delta\left(a_{i}, b_{i}\right)$ for all $i \in I$ such that $a^{\prime} \searrow b^{\prime} \sqsubseteq \bigsqcup_{i \in I} a_{i} \searrow b_{i}$. From Proposition 3.13, it follows that $\delta\left(a^{\prime}, b^{\prime}\right) \supseteq \bigcap_{i \in I} \delta\left(a_{i}, b_{i}\right)$ and thus $f \in \delta\left(a^{\prime}, b^{\prime}\right)$ as required.

Note the significance of Theorem 4.2. In classical mathematics, the derivative of a continuous function $f:[0,1] \rightarrow \mathbb{R}$ at a point $x \in[0,1]$ may not exist, and, in fact, $f$ may be nowhere differentiable; even if the derivative $f^{\prime}(x)$ exists at all points $x \in[0,1]$, the map $x \mapsto f^{\prime}(x):[0,1] \rightarrow \mathbb{R}$ may not be continuous. Such 'irregularities' do not happen in the domain-theoretic world. In fact, any Scott continuous function $f: \mathbf{I}[0,1] \rightarrow \mathbf{I} \mathbb{R}$ is differentiable and its derivative $\frac{d f}{d x}: \mathbf{I}[0,1] \rightarrow \mathbf{I} \mathbb{R}$ is a Scott continuous map, that is, has the same type as $f$ itself.

\section{Proposition 4.3.}

(i) Let $f: \mathbf{I}[0,1] \rightarrow \mathbf{I} \mathbb{R}$ be Scott continuous. Suppose for some $z \in[0,1], f(\{z\})$ is not maximal, then $\frac{d f}{d x}(\{z\})=\perp$.

(ii) Suppose $f \in D^{0}$. If $\frac{d f}{d x}(\{y\})=\{c\}$ is maximal, then $f$ sends maximal elements to maximal elements in a neighbourhood $U$ of $y$ and the derivative of the induced restriction $f: U \rightarrow \mathbb{R}$ exists at $y$ and $f^{\prime}(y)=c$. 
(iii) If $g:[0,1] \rightarrow \mathbb{R}$ is a bounded, integrable function, and $f:[0,1] \rightarrow \mathbb{R}$ is a (classical) indefinite integral of $g$, that is, $f(y)=\int_{0}^{y} g d \mu$, then $\underline{\lim } g(y) \leqslant \underline{\lim } \frac{f(x)-f(y)}{x-y}$, $\varlimsup \frac{f(x)-f(y)}{x-y} \leqslant \varlimsup \lim g(y)$ and $\frac{d \mathbf{I} f}{d x}(\{y\}) \sqsupseteq[\lim g(y), \varlimsup \lim g(y)]$.

\section{Proof.}

(i) By Proposition 3.2, for $b \neq \perp$, we have $f \in \delta(a, b) \Rightarrow z \notin a^{\circ}$. Hence,

$$
\frac{d f}{d x}(\{z\})=\bigsqcup\left\{b \mid f \in \delta(a, b) \& z \in a^{\circ}\right\}=\perp .
$$

(ii) We have $\{c\}=\frac{d f}{d x}(\{y\})=\bigsqcup\left\{b \mid y \in a^{\circ} \& f \in \delta(a, b)\right\}$. For any neighbourhood $O$ of $c$ in $\mathbb{R}$, there exists $b$ with $c \in b^{\circ}$ and $b \subset O$ such that there exists $a \in \mathbf{I}[0,1]$ with $y \in a$ and $f \in \delta(a, b)$. By Proposition 3.2(i), this implies first that $f(\{x\})$ is maximal for each $x \in a^{\circ}$ and second that the restriction $f: a^{\circ} \rightarrow \mathbb{R}$ satisfies $\frac{f(x)-f(y)}{x-y} \in b \subseteq O$ for all $x \in a^{\circ}$ with $x \neq y$, that is, $\lim _{x \rightarrow y} \frac{f(x)-f(y)}{x-y}=c$.

(iii) Let $\epsilon>0$ be given. There exists a neighbourhood $(\underline{a}, \bar{a})$ of $y$ such that for all $x \in a^{\circ}$ we have

$$
-\epsilon+\underline{\lim } g(y) \leqslant g(x) \leqslant \epsilon+\varlimsup \lim g(y) .
$$

Integration on $[x, z]$ yields

$$
(-\epsilon+\underline{\lim } g(y))(z-x) \leqslant f(z)-f(x) \leqslant(\epsilon+\varlimsup \lim g(y))(z-x) .
$$

Thus, $\mathbf{I} f \in \delta(a,[-\epsilon+\underline{\lim } g(y), \epsilon+\overline{\lim } g(y)])$ and the first two inequalities follow. Since $\epsilon>0$ is arbitrary, we also get

$$
\frac{d \mathbf{I} f}{d x}(\{y\})=\bigsqcup\left\{b \mid y \in a^{\circ}, \mathbf{I} f \in \delta(a, b)\right\} \sqsupseteq[\lim g(y), \overline{\lim } g(y)] .
$$

Example 4.4. The derivatives of the canonical extensions of $f_{1}, f_{2}, f_{3}$ and $g$ of Example 3.3 are given by

$$
\begin{aligned}
& \frac{d \mathbf{I} f_{1}}{d x}(y)=\left\{\begin{array}{ll}
\{-1\} & \text { if } \bar{y}<0 \\
\{1\} & \text { if } \underline{y}>0 \\
{[-1,1]} & \text { if } 0 \in y
\end{array} \quad \frac{d \mathbf{I} f_{2}}{d x}(y)= \begin{cases}\mathbf{I} f^{\prime}(y) & \text { if } 0 \notin y \\
{[-1,1]} & \text { if } 0 \in y\end{cases} \right. \\
& \frac{d \mathbf{I} f_{3}}{d x}(y)=\left\{\begin{array}{ll}
\mathbf{I} f^{\prime}(y) & \text { if } 0 \notin y \\
\perp & \text { if } 0 \in y
\end{array} \quad \frac{d \mathbf{I} g}{d x}(y)= \begin{cases}\{0\} & \text { if } 1 / 2 \notin y \\
\perp & \text { if } 1 / 2 \in y .\end{cases} \right.
\end{aligned}
$$

Note that the classical derivative of $f_{2}$ exists at 0 but the derivative is not continuous at 0 .

The operator

$$
\begin{aligned}
\frac{d}{d x}: D^{0}[0,1] & \rightarrow D^{0}[0,1] \\
f & \mapsto \frac{d f}{d x}
\end{aligned}
$$

is easily seen to be monotone but not continuous. In fact, the derivative of any step function $\bigsqcup_{i \in I} a_{i} \searrow b_{i}$ for intervals $b_{i}(i \in I)$ with non-zero length is the bottom function. Since, for example, the function $\mathbf{I} f$, where $f=0$ is a constant function, can be constructed 
as the lub of an increasing chain of such step functions, it follows that $\frac{d}{d x}$ is not continuous. In Section 6, we will define what we will call the continuous derivative operator. Finally, we note that the definition of the derivative together with Theorem 4.2 and Proposition 4.3 easily extend to $D_{\mathrm{r}}^{0}$.

We finally obtain the generalisation of Theorem 4.2(iii) to ties, which establishes a duality between primitive maps and differentiation.

Corollary 4.5. $f \in \int g$ iff $g \sqsubseteq \frac{d f}{d x}$.

Proof. Let $g=\bigsqcup_{i \in I} a_{i} \searrow b_{i}$. Then, by Theorem 4.2(iii),

$$
f \in \int g \Longleftrightarrow f \in \bigcap_{i \in I} \delta\left(a_{i}, b_{i}\right) \Longleftrightarrow \forall i \in I . a_{i} \searrow b_{i} \sqsubseteq \frac{d f}{d x} \Longleftrightarrow \bigsqcup_{i \in I} a_{i} \searrow b_{i} \sqsubseteq \frac{d f}{d x} .
$$

The above duality can be considered as a fundamental theorem of calculus for intervalvalued functions of an interval variable. In the next section, we will see how a variant of this result for functions of a real variable is closely related to the classical fundamental theorem of calculus.

\section{Functions of a real variable}

Having studied ties of $D^{0}$, we now consider the similar notion for $D_{\mathrm{r}}^{0}$. We will derive an interval version of the fundamental theorem of calculus and establish the relation between the domain theoretic derivative and the Clarke's derivative. Furthermore, the information ordering for the ties of $D_{\mathrm{r}}^{0}$ has a simple characterization, which we will determine here.

The single-step tie $\delta_{\mathrm{r}}(a, b) \subset D_{\mathrm{r}}^{0}$ is simply the restriction of $\delta(a, b)$ to the subdomain $D_{\mathrm{r}}^{0}$, in other words $\delta_{\mathrm{r}}(a, b)=\mathscr{I}\left(\delta(a, b) \cap \mathscr{E}\left(D_{\mathrm{r}}^{0}\right)\right)$; more generally, a tie of $D_{\mathrm{r}}^{0}$ is given by $\bigcap_{i \in I} \delta_{\mathrm{r}}\left(a_{i}, b_{i}\right)=\mathscr{I}\left(\left(\bigcap_{i \in I} \delta\left(a_{i}, b_{i}\right)\right) \cap \mathscr{E}\left(D_{\mathrm{r}}^{0}\right)\right)$ for any tie $\bigcap_{i \in I} \delta\left(a_{i}, b_{i}\right)$ of $D^{0}$. Notice that, by Proposition 3.2(i), any step tie of $D_{\mathrm{r}}^{0}$ is essentially a collection of classical Lipschitz functions. In particular, for $f \in D_{\mathrm{r}}^{0}$ and $b \neq \perp$, we have $f \in \delta_{\mathrm{r}}(a, b)$ iff the image of $a^{\circ}$ under $f$ is a subset of maximal elements of $\mathbf{I R}$ and the induced map $f: a^{\circ} \rightarrow \mathbb{R}$ satisfies $\underline{b}(x-y) \leqslant f(x)-f(y) \leqslant \bar{b}(x-y)$ for all $x \geqslant y$ with $x, y \in a^{\circ}$. Clearly, for $a^{\circ} \neq \varnothing$ and $b \neq \perp$, we have $\delta_{\mathrm{r}}(a, b) \supseteq \delta_{\mathrm{r}}(c, d)$ iff $a \subseteq c$ and $d \subseteq b$.

\section{Proposition 5.1.}

(i) $f \in \bigcap_{i \in I} \delta_{\mathrm{r}}\left(a_{i}, b_{i}\right) \Longleftrightarrow \mathscr{E}(f) \in \bigcap_{i \in I} \delta\left(a_{i}, b_{i}\right)$.

(ii) $\mathscr{I}(f) \in \bigcap_{i \in I} \delta_{\mathrm{r}}\left(a_{i}, b_{i}\right)$ if $f \in \bigcap_{i \in I} \delta\left(a_{i}, b_{i}\right)$.

Proof.

(i) We have $f \in \bigcap_{i \in I} \delta_{\mathrm{r}}\left(a_{i}, b_{i}\right)$ iff $f=\mathscr{I}(g)$ for some $g \in \bigcap_{i \in I} \delta\left(a_{i}, b_{i}\right) \cap \mathscr{E}\left(D_{\mathrm{r}}^{0}\right)$ iff $f=\mathscr{I}(g)$ and $g \in \bigcap_{i \in I} \delta\left(a_{i}, b_{i}\right) \cap \mathscr{E}\left(D_{\mathrm{r}}^{0}\right)$ with $g=\mathscr{E}(h)$ for some $h \in D_{\mathrm{r}}^{0}$. Since $\mathscr{I} \circ \mathscr{E}=1_{D_{\mathrm{r}}^{0}}$, the latter condition gives $h=f$ and the result follows.

(ii) If $f \in \bigcap_{i \in I} \delta\left(a_{i}, b_{i}\right)$, then $\mathscr{E} \circ \mathscr{I}(f) \in \bigcap_{i \in I} \delta\left(a_{i}, b_{i}\right)$ since ties are upper sets. The result follows by (i).

Proposition 5.2. For any indexing set $I$, we have $\bigcap_{i \in I} \delta_{\mathrm{r}}\left(a_{i}, b_{i}\right) \neq \varnothing$ iff the family of step functions $\left(a_{i} \searrow_{\mathrm{r}} b_{i}\right)_{i \in I}$ is consistent. 
Proof. This follows immediately by Propositions 3.11 and 5.1(i) together with Equation 1.

We also have the following counterparts of Proposition 3.11, Corollary 3.14 and Proposition 3.15 for ties in $D_{\mathrm{r}}^{0}$.

Proposition 5.3. $\delta_{\mathrm{r}}(a, b) \supseteq \bigcap_{i \in I} \delta_{\mathrm{r}}\left(a_{i}, b_{i}\right)$ if $a \searrow_{\mathrm{r}} b \sqsubseteq \bigsqcup_{i \in I} a_{i} \searrow_{\mathrm{r}} b_{i}$.

Proof. This can be proved by the same method used to prove Proposition 3.11.

Corollary 5.4. $\bigcap_{i \in I} \delta_{\mathrm{r}}\left(a_{i}, b_{i}\right) \supseteq \bigcap_{i \in J} \delta_{\mathrm{r}}\left(a_{i}, b_{i}\right)$ if $\bigsqcup_{i \in I} a_{i} \searrow_{\mathrm{r}} b_{i} \sqsubseteq \bigsqcup_{i \in J} a_{i} \searrow_{\mathrm{r}} b_{i}$.

Let $\left(T_{\mathrm{r}}^{1}[0,1], \supseteq\right)$ be the poset of ties of $D_{\mathrm{r}}^{0}$ ordered by reverse inclusion.

Proposition 5.5. $\left(T_{\mathrm{r}}^{1}[0,1], \supseteq\right)$ is a dcpo.

Furthermore, the integral map $\int_{\mathrm{r}}: D_{\mathrm{r}}^{0} \rightarrow T_{\mathrm{r}}^{1}$ defined by $\int_{\mathrm{r}} \bigsqcup_{i \in I} a_{i} \searrow_{\mathrm{r}} b_{i}=\bigcap_{i \in I} \delta_{\mathrm{r}}\left(a_{i}, b_{i}\right)$ is well-defined, onto and continuous; it satisfies $\int_{\mathrm{r}}=\mathscr{I} \circ \int \circ \mathscr{E}$. For convenience we write $\int$ for $\int_{r}$ when no ambiguity can arise.

\subsection{Fundamental theorem of calculus}

As in the case of functions with interval input, we can define the domain-theoretic derivative of a function of a real variable.

Definition 5.6. The derivative of a continuous function $f:[0,1] \rightarrow \mathbb{I} \mathbb{R}$ is the map

$$
\frac{d f}{d x}=\bigsqcup_{f \in \delta_{\mathrm{r}}(a, b)} a \searrow_{\mathrm{r}} b:[0,1] \rightarrow \mathbf{I} \mathbb{R} .
$$

Given $f \in C^{0}[0,1]$, we write if for the map if $:[0,1] \rightarrow \operatorname{IIR}$ given by $\mathbf{i} f(x)=\{f(x)\}$. As in Theorem 4.2, we obtain the following theorem.

\section{Theorem 5.7.}

(i) $\frac{d f}{d x}$ is well-defined and Scott continuous.

(ii) If $f \in C^{1}[0,1]$, then $\frac{d \mathbf{i} f}{d x}=\mathbf{i} f^{\prime}$.

(iii) $f \in \delta_{\mathrm{r}}(a, b)$ iff $a \searrow_{\mathrm{r}} b \sqsubseteq \frac{d f}{d x}$.

We then have the following counterpart of Corollary 4.5.

Corollary 5.8. For $f, g \in D_{\mathrm{r}}^{0}$, we have $f \in \int g$ iff $g \sqsubseteq \frac{d f}{d x}$.

By restricting to continuous classical functions, we obtain the classical fundamental theorem of calculus.

Corollary 5.9. If $f, g \in D_{\mathrm{r}}^{0}$ are extensions of classical $C^{0}$ function, we have $f \in \int g$ iff $g=\frac{d f}{d x}$.

It follows from Corollary 5.9, that the duality of differentiation and primitive functions in Corollary 5.8 can be considered as an extension of the classical fundamental theorem of calculus to interval-valued functions of a real variable. 


\subsection{Relation with Clarke's' derivative}

We now recall the notion of Clarke's derivative for Lipschitz functions. Recall that any function $f:[0,1] \rightarrow \mathbb{R}$ that is Lipschitz in a neighbourhood of a point, is differentiable almost everywhere in that neighbourhood. We use $\Omega_{f} \subset[0,1]$ to denote the set of points at which $f$ is not differentiable.

Definition 5.10 (Clarke (1983, page 63)). Let $f:[0,1] \rightarrow \mathbb{R}$ be Lipschitz near $x \in[0,1]$. Then the Clarke's derivative of $f$ at $x$ is defined as

$$
\partial f=\operatorname{co}\left\{\lim f^{\prime}\left(x_{m}\right): x_{m} \rightarrow x, x_{m} \notin \Omega_{f}\right\},
$$

where for a subset $A \subset \mathbb{R}$ the convex set generated by $A$ is denoted by $\operatorname{co} A$.

The meaning of the above expression is as follows: consider any sequence $x_{m} \in[0,1]^{n} \backslash \boldsymbol{\Omega}_{f}$, $m \in \mathbb{N}$, tending to $x$ such that the sequence $f^{\prime}\left(x_{m}\right)$ converges, then the convex hull of all such limits is the Clarke's derivative of $f$ at $x$.

We note that the two notions of Clarke's derivative and the domain-theoretic derivative both extend to functions of several variables: $f:[0,1]^{n} \rightarrow \mathbb{R}$. The Clarke's derivative in higher dimensions is usually referred to as Clarke's gradient. The Clarke's gradient of a function at a point where the function is locally Lipschitz is a non-empty, compact and convex subset of $\mathbb{R}^{n}$ (Clarke 1983, page 27). We now establish the connection between the domain-theoretic derivative and the Clarke's derivative.

Theorem 5.11. For any function $f:[0,1] \rightarrow \mathbb{R}$, the domain-theoretic derivative at a point where the function is locally Lipschitz coincides with the Clarke's derivative at that point.

Proof. This follows from a more general result (Edalat et al. 2004, Theorem 4.2) relating the domain-theoretic derivative of a real-valued locally Lipschitz function of $n$ real variables with the Clarke's gradient: the domain-theoretic derivative gives the smallest $n$-dimensional rectangle containing the Clarke's gradient. The result then follows since in dimension one the Clarke gradient, that is, the Clarke derivative, is a non-empty compact interval.

\subsection{Partial order of ties}

The information order in $T_{\mathrm{r}}^{1}$ has an elementary characterisation, which we will now determine. Note that the result of this subsection is not used in the rest of the paper and you may wish to skip it in the first reading. Define the function

$$
r: D_{\mathrm{r}}^{0} \rightarrow\left([0,1]^{2} \rightarrow \mathbf{I I R}\right)
$$

by

and

$$
r^{-}(g):(x, y) \mapsto \begin{cases}\int_{x}^{y} g^{-} d \mu & x, y \text { in the same component of } \operatorname{dom}(g) \\ \perp & \text { otherwise, }\end{cases}
$$

$$
r^{+}(g):(x, y) \mapsto \begin{cases}\int_{x}^{y} g^{+} d \mu & x, y \text { in the same component of } \operatorname{dom}(g) \\ \perp & \text { otherwise. }\end{cases}
$$


By the monotone convergence theorem, $r$ is a Scott continuous function. We also define the Scott continuous map $[s, t]: D_{\mathrm{r}}^{0} \rightarrow\left([0,1]^{2} \rightarrow \mathbf{I R}\right)$ by

$$
\begin{aligned}
& s(g):(x, y) \mapsto \begin{cases}r^{-}(g)(x, y) & \text { if } x \leqslant y \text { with } x, y \text { in the same component of } \operatorname{dom}(g) \\
-r^{+}(g)(y, x) & \text { if } y \leqslant x \text { with } x, y \text { in the same component of } \operatorname{dom}(g) \\
\perp & \text { otherwise, }\end{cases} \\
& t(g):(x, y) \mapsto \begin{cases}r^{+}(g)(x, y) & \text { if } x \leqslant y \text { with } x, y \text { in the same component of } \operatorname{dom}(g) \\
-r^{-}(g)(y, x) & \text { if } y \leqslant x \text { with } x, y \text { in the same component of } \operatorname{dom}(g) \\
\perp & \text { otherwise. }\end{cases}
\end{aligned}
$$

\section{Proposition 5.12.}

(i) Let $\Delta \in T_{\mathrm{r}}^{1}$ and consider any $g \in D_{\mathrm{r}}^{0}$ with $\Delta=\int g$. Then $\lambda y \cdot s(g)(x, y)$ and $\lambda y \cdot t(g)(x, y)$ are in $\Delta$, both vanishing at $x$. Moreover, we have

$$
h \in \Delta \Longleftrightarrow r^{-}(g)(x, y) \leqslant h(y)-h(x) \leqslant r^{+}(g)(x, y)
$$

for all $x, y$ belonging to the same component of $\operatorname{dom}(g)$ with $x \leqslant y$.

(ii) The functions $\lambda y \cdot s(g)(x, y)$ and $\lambda y \cdot t(g)(x, y)$ are, respectively, the least and greatest functions $h \in \Delta$ with $h(x)=0$.

(iii) The following are equivalent:

$-\int g_{1} \supseteq \int g_{2}$

$-g_{1}^{-} \leqslant g_{2}^{-}$a.e. and $g_{2}^{+} \leqslant g_{1}^{+}$a.e.,

- for all $x, y$ with $x \leqslant y$ we have $r^{-}\left(g_{1}\right)(x, y) \leqslant r^{-}\left(g_{2}\right)(x, y)$ and $r^{+}\left(g_{2}\right)(x, y) \leqslant$ $r^{+}\left(g_{1}\right)(x, y)$,

$-s\left(g_{1}\right) \leqslant s\left(g_{2}\right)$ and $t\left(g_{1}\right) \geqslant t\left(g_{2}\right)$.

(iv) $\int g_{1}=\int g_{2}$ iff $g_{1}^{-}=g_{2}^{-}$a.e. and $g_{1}^{+}=g_{2}^{+}$a.e.

(v) $\delta_{\mathrm{r}}(a, b) \ll \bigcap_{i \in I} \delta_{\mathrm{r}}\left(a_{i}, b_{i}\right)$ if $a \searrow_{\mathrm{r}} b \ll \bigsqcup_{i \in I} a_{i} \searrow_{\mathrm{r}} b_{i}$.

Proof.

(i) Let $g=\bigsqcup_{i \in I} a_{i} \searrow b_{i}$ with $\int g=\Delta$. Take $x, y$ in the same component of $\operatorname{dom}(g)$ with $x \leqslant y$. Note that if $h \in \Delta$, then, being locally Lipschitz (and hence absolutely continuous) in $\operatorname{dom}(\Delta)=\operatorname{dom}(g), h$ is differentiable a.e. (with respect to the Lebesgue measure) in $\operatorname{dom}(g)$, and $h$ is equal to the integral of its derivative, that is, $h(y)-h(x)=$ $\int_{x}^{y} h^{\prime} d \mu$ (Hewitt and Stromberg 1975, Sections 17.17 and 18.17). In the following, whenever we write $h^{\prime}(x)$ in a relation, we mean that the relation holds whenever $h^{\prime}(x)$ exists, which is a.e. We have $h \in \bigcap_{i \in I} \delta_{\mathrm{r}}\left(a_{i}, b_{i}\right)$ iff $\forall i \in I, x \in a_{i}^{\circ} . \underline{b}_{i} \leqslant h^{\prime}(x) \leqslant \overline{b_{i}}$ iff $\sup _{x \in a_{i}^{\circ}} \underline{b_{i}} \leqslant h^{\prime}(x) \leqslant \inf _{x \in a_{i}^{\circ}} \overline{b_{i}}$ iff $h^{\prime}(x) \in \bigsqcup_{x \in a_{i}^{\circ}} b_{i}$ iff $\forall x \in \operatorname{dom}(g) \cdot g^{-}(x) \leqslant h^{\prime}(x) \leqslant$ $g^{+}(x)$ iff $\int_{x}^{y} g^{-} d \mu \leqslant h(y)-h(x) \leqslant \int_{x}^{y} g^{+} d \mu$, for all $x, y$ belonging to the same component of $\operatorname{dom}(g)$ with $x \leqslant y$.

(ii) By part (i), any $h \in \Delta$ with $h(x)=0$ satisfies $s(g)(x, y) \leqslant h(y) \leqslant t(g)(x, y)$ for all $x, y$ in the same component of $\operatorname{dom}(g)$. On the other hand, by Lemma 3.10, the maps $\lambda y . s(g)(x, y)$ and $\lambda y \cdot t(g)(x, y)$ belong to $\Delta$ for any $x \in \operatorname{dom}(g)$. 
(iii) This follows from parts (i) and (ii).

(iv) This is an immediate consequence of part (iii).

(v) Assume $a \searrow_{\mathrm{r}} b \ll \bigsqcup_{i \in I} a_{i} \searrow_{\mathrm{r}} b_{i}$, that is, $\bigsqcup_{a_{i} \ll a} b_{i} \gg b$. Let $\left(\Delta_{i}\right)_{i \in J}$ be a directed set of ties with $\bigcap_{i \in I} \delta_{\mathrm{r}}\left(a_{i}, b_{i}\right) \supseteq \bigcap_{i \in J} \Delta_{i}$. Take $g_{i} \in D_{\mathrm{r}}^{0}$ with $\Delta_{i}=\int g_{i}$ for all $i \in J$ and let $g=\bigsqcup_{i \in I} a_{i} \searrow_{\mathrm{r}} b_{i}$. From $a \searrow_{\mathrm{r}} b \ll g$ we get $b \ll g(a)$. Put $c=g(a)$, so $\underline{b}<\underline{c}$ and $\bar{c}<\bar{b}$. Then, for all $x, y, z \in a$ with $y \geqslant z \geqslant x$ we have $\underline{c} \leqslant g^{-}(z)$ and $g^{+}(z) \leqslant \bar{c}$. Integrating from $x$ to $y$, by part (i) we get

$$
\begin{aligned}
& \underline{b}(y-x)<\underline{c}(y-x) \leqslant s(g)(x, y) \leqslant \sup _{i \in J} s\left(g_{i}\right)(x, y) \\
& \inf _{i \in J} t\left(g_{i}\right)(x, y) \leqslant t(g)(x, y) \leqslant \bar{c}(y-x)<\bar{b}(y-x) .
\end{aligned}
$$

Thus, for the directed set of continuous maps $\left(\lambda x . \lambda y \cdot s\left(g_{i}\right)(x, y)-\underline{b}(y-x)\right)_{i \in J}$, we have

$$
\left.\sup _{i \in J}\left(\lambda x . \lambda y . s\left(g_{i}\right)(x, y)-\underline{b}(y-x)\right) \geqslant s(g(x, y))-\underline{b}(y-x)\right)>0 .
$$

It follows that the directed family of continuous functions

$$
\left(\lambda x \cdot \lambda y \cdot \min \left(s\left(g_{i}(x, y)\right)-\underline{b}(y-x), s(g)-\underline{b}(y-x)\right)\right)_{i \in J}
$$

converges monotonically, thus uniformly, to the function $\lambda x . \lambda y \cdot s(g)-\underline{b}(y-x)>0$ on the compact set $\left\{(x, y) \in[0,1]^{2} \mid x \geqslant y\right\}$. Therefore, there exists $i \in J$ such that $\left.\lambda x \cdot \lambda y \cdot \min \left(s\left(g_{i}(x, y)\right)-\underline{b}(y-x), s(g)-\underline{b}(y-x)\right)\right)>0$ and thus $\lambda x . \lambda y \cdot s\left(g_{i}(x, y)\right)-\underline{b}(y-x)$ $>0$. Similarly, there exists $i \in J$ such that $\lambda x . \lambda y \cdot t\left(g_{i}(x, y)\right)-\underline{b}(y-x)<0$. Hence there exists $i \in J$ with $\lambda x . \lambda y \cdot s\left(g_{i}(x, y)\right)-\underline{b}(y-x)>0$ and $\lambda x . \lambda y \cdot t\left(g_{i}(x, y)\right)-\underline{b}(y-x)<0$. It follows, by part (i) again, that $\delta(a, b) \supseteq \Delta_{i}$, as required.

The characterisation of the information ordering for $T^{1}$ is far more involved than Proposition 5.12 for $T_{\mathrm{r}}^{1}$; we will present it in a future paper.

\section{The Domain of $C^{1}$ functions}

We now define the domain of $C^{1}$ functions. Consider the relation Cons $\subset D^{0} \times D^{0}$ defined by $(f, g) \in$ Cons if $\uparrow f \cap \int g \neq \varnothing$. We will show that this relation is Scott closed and decidable on basis elements.

Proposition 6.1. Let $g \in D^{0}$ and $\left(f_{i}\right)_{i \in I}$ be a non-empty family of functions $f_{i}: \operatorname{dom}(g) \rightarrow$ $\mathbb{R}$ with $\mathbf{I} f_{i} \in \int g$ for all $i \in I$. If $h_{1}=\inf _{i \in I} f_{i}$ is real-valued, then $\mathbf{I} h_{1} \in \int g$. Similarly, if $h_{2}=\sup _{i \in I} f_{i}$ is real-valued, then $\mathbf{I} h_{2} \in \int g$.

Proof. Suppose $h_{1}$ is real-valued. Let $a \searrow b \sqsubseteq g$. From Proposition 3.2, we have $\underline{b}(u-v) \leqslant$ $f_{i}(u)-f_{i}(v) \leqslant \bar{b}(u-v)$ for all $u, v \in a^{\circ}$ with $u>v$ and all $i \in I$. Taking infimum, we get $\underline{b}(u-v) \leqslant h_{1}(u)-h_{1}(v) \leqslant \bar{b}(u-v)$ and, by Proposition 3.2, $\mathbf{I} h_{1} \in \delta(a, b)$, as required. The case for $h_{2}$ is similar.

Let $L[0,1]$ be the set of partial maps of $[0,1]$ into the extended real line. Consider the two dcpo's $(L[0,1], \leqslant)$ and $(L[0,1], \geqslant)$. Define the maps $s: D^{0} \times D^{0} \rightarrow(L, \leqslant)$ and 


$$
\begin{aligned}
t: D^{0} \times D^{0} \rightarrow(L, \geqslant) \text { by } & \\
s:(f, g) & \mapsto \inf \left\{h: \operatorname{dom}(g) \rightarrow \mathbb{R} \mid \mathbf{I} h \in \int g \& h \geqslant f^{-}\right\} \\
t & :(f, g) \mapsto \sup \left\{h: \operatorname{dom}(g) \rightarrow \mathbb{R} \mid \mathbf{I} h \in \int g \& h \leqslant f^{+}\right\} .
\end{aligned}
$$

We use the convention that the infimum and supremum of the empty set are $\infty$ and $-\infty$, respectively. Note that given a connected component $A$ of $\operatorname{dom}(g)$ with $A \cap \operatorname{dom}(f)=\varnothing$, then $s(f, g)(x)=-\infty$ and $t(s, f)(x)=\infty$ for $x \in A$.

Proposition 6.2. The following are equivalent:

(i) $(f, g) \in$ Cons.

(ii) $s(f, g) \leqslant t(f, g)$.

(iii) There exists $h: \operatorname{dom}(g) \rightarrow \mathbb{R}$ with $\mathbf{I} h \in \int g$ and $f^{-} \leqslant h \leqslant f^{+}$on $\operatorname{dom}(g)$.

Proof. If $\operatorname{dom}(f) \cap \operatorname{dom}(g)=\varnothing$, the three statements hold trivially. So assume in the following proof that $\operatorname{dom}(f) \cap \operatorname{dom}(g) \neq \varnothing$.

(ii) $\Rightarrow$ (i). Suppose $s(f, g) \leqslant t(f, g)$. Then, $f \sqcup \mathbf{I} s(f, g) \in \uparrow f \cap \int g$ and hence $(f, g) \in$ Cons.

(i) $\Rightarrow$ (ii). Suppose $(f, g) \in$ Cons. Assume $h \in \uparrow f \cap \int g$. Then, the induced map $h$ : $\operatorname{dom}(g) \rightarrow \mathbb{R}$ satisfies $\mathbf{I} h \in \int g$. Hence, $f^{-} \leqslant h \leqslant f^{+}$and thus $s(f, g) \leqslant t(f, g)$.

(ii) $\Rightarrow$ (iii). Suppose $s(f, g) \leqslant t(f, g)$. Put $h=s(f, g)$.

(iii) $\Rightarrow$ (ii). We have $s(f, g) \leqslant h \leqslant t(f, g)$.

Proposition 6.3. The maps $s$ and $t$ are Scott continuous.

Proof. Consider the map s. If $f_{1} \sqsubseteq f_{2}$ and $g_{1} \sqsubseteq g_{2}$, we have $\int g_{1} \supseteq \int g_{2}$ and $f_{1}^{-} \leqslant f_{2}^{-}$, and it follows that $s\left(f_{1}, g_{1}\right) \leqslant s\left(f_{2}, g_{2}\right)$. Let $\left\{\left(f_{i}, g_{i}\right)\right\}_{i \in \omega}$ be an increasing chain and put $f=\bigsqcup_{i \in \omega} f_{i}$ and $g=\bigsqcup_{i \in \omega} g_{i}$. To show the continuity of $s$, we need to show that $\sup _{i} s\left(f_{i}, g_{i}\right) \geqslant s(f, g)$ on any connected component of $\operatorname{dom}(g)=\bigcup_{i \in \omega} \operatorname{dom}\left(g_{i}\right)$. Take any such connected component $A \subseteq \operatorname{dom}(g)$. If $A \cap \operatorname{dom}(f)=\varnothing$, then $s(f, g)=-\infty$ on $A$ and the result follows. Assume that $A \cap \operatorname{dom}(f) \neq \varnothing$, that is, $\operatorname{dom} f_{i_{0}} \cap \operatorname{dom} g_{i_{0}} \neq \varnothing$ for some $i_{0} \in \omega$. If $s\left(f_{i}, g_{i}\right)=\infty$ on $A \cap \operatorname{dom}\left(g_{i}\right)$ for some $i \geqslant i_{0}$, then $\sup _{i \in \omega} s\left(f_{i}, g_{i}\right)=\infty$ on $A$ and the result again follows. Otherwise, assume without loss of generality that $-\infty<s\left(f_{i}, g_{i}\right)<\infty$ on $A \cap \operatorname{dom}\left(g_{i}\right)$ for all $i \in \omega$. Then, from $\left.\mathbf{I}\left(s\left(f_{i}, g_{i}\right)\right)\right|_{A} \in \int g_{i}$, it follows that $\forall i \geqslant j . \mathbf{I}\left(s\left(f_{i}, g_{i}\right)\right) \uparrow_{A} \in \int g_{j}$, and hence, by Proposition 6.1, $\mathbf{I}\left(\sup _{i \in \omega}\left(s\left(f_{i}, g_{i}\right) \uparrow_{A}\right)\right) \in \int g_{j}$. Thus $\left.\mathbf{I}\left(\sup _{i \in \omega} s\left(f_{i}, g_{i}\right)\right)\right|_{A} \in \bigsqcup_{j} \int g_{j}=\int \bigsqcup g_{j}$. On the other hand, $s\left(f_{i}, g_{i}\right) \geqslant f_{i}^{-}$on $A$ implies $\sup _{i \in \omega} s\left(f_{i}, g_{i}\right) \geqslant f_{i}^{-}$on $A$ and hence $\sup _{i \in \omega} s\left(f_{i}, g_{i}\right) \geqslant f^{-}$on $A$. This shows that $s$ is continuous. By similar reasoning, $t$ is continuous.

Corollary 6.4. The relation Cons is Scott closed.

Proof. Let $\left(f_{i}, g_{i}\right)_{i \in I} \subset D^{0} \times D^{0}$ be a directed family with $\left(f_{i}, g_{i}\right) \in$ Cons for all $i \in I$. Then, by Proposition $6.2, s\left(f_{i}, g_{i}\right) \leqslant t\left(f_{i}, g_{i}\right)$ for all $i \in I$. Hence, $s(f, g)=\sup _{i \in I} s\left(f_{i}, g_{i}\right) \leqslant$ $\inf _{i \in I} t\left(f_{i}, g_{i}\right)=t(f, g)$.

Corollary 6.5. Let $(f, g) \in$ Cons. Then in each connected component $O$ of the domain of definition of $g$ that intersects the domain of definition of $f$, there exist two locally 

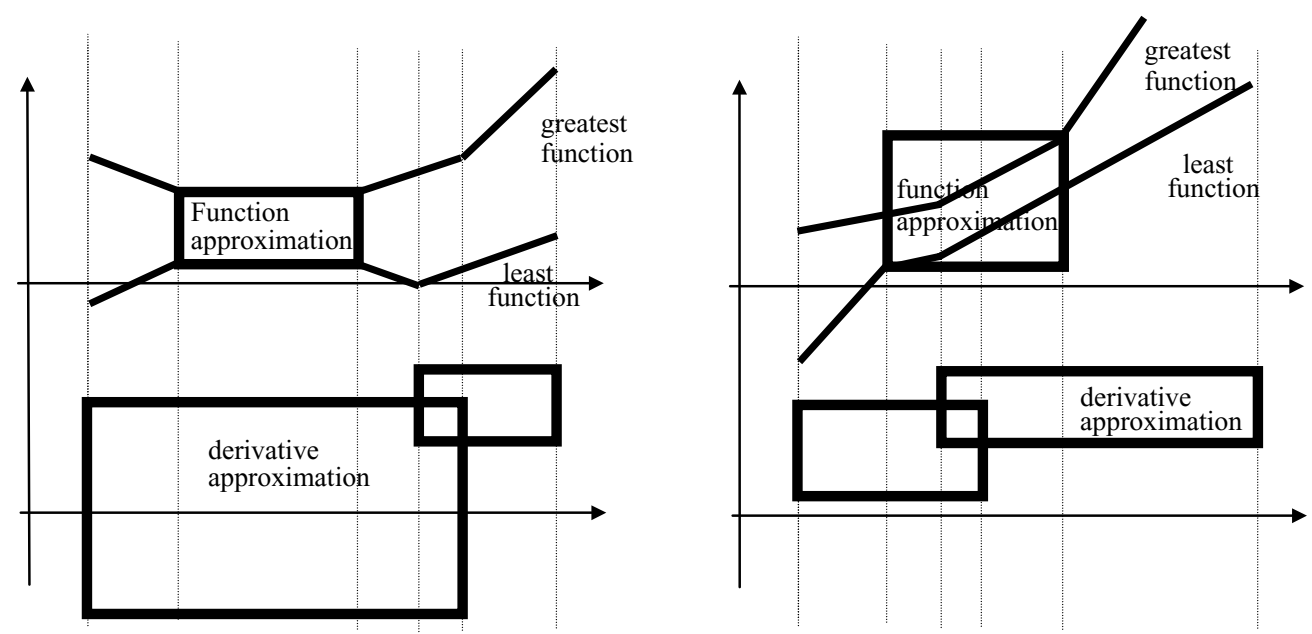

Fig. 2. Two pairs of functions in Cons

Lipschitz functions $s: O \rightarrow \mathbb{R}$ and $t: O \rightarrow \mathbb{R}$ such that $\mathbf{I} s, \mathbf{I} t \in \uparrow f \cap \int g$, and for each $u \in \uparrow f \cap \int g$, we have $u(\{x\})=\{v(x)\}$ with $s(x) \leqslant v(x) \leqslant t(x)$ for all $x \in O$ (see Figure 2).

Next we will show that Cons is decidable on (rational or semi-rational polynomial) basis elements. To construct an effective structure for $D^{1}$ later in this section, only the decidability of consistency on rational step functions is required and the reader may skip the details of the following lemma for the general case of rational or semi-rational polynomial basis in the first reading and assume that the basis elements $(f, g)$ in the lemma are rational step functions, in which case the proof is considerably simpler. We note the following general property.

Proposition 6.6. If $\alpha, \beta:[v, w] \rightarrow \mathbb{R}$ are two piecewise semi-rational polynomials, then the relation $\alpha \leqslant \beta$ (that is, $\forall x \in[v, w] . \alpha(x) \leqslant \beta(x)$ ) is decidable.

Lemma 6.7. Let $f, g \in D^{0}[0,1]$ be semi-rational basis elements with $(f, g) \in$ Cons. Then the functions $s(f, g)$ and $t(f, g)$ are piecewise semi-rational polynomials in each connected component of $\operatorname{dom}(g)$ with non-empty intersection with $\operatorname{dom}(f)$.

Proof. Fix a connected component $O=(v, w)$ of $\operatorname{dom}(g)$ with $O \cap \operatorname{dom}(f) \neq \varnothing$. Then, for $x \in O$, we have $s(f, g)(x)=\sup _{y \in O \cap \operatorname{dom}(f)} S_{(f, g)}(x, y)$ where $S_{(f, g)}: O \times(O \cap \operatorname{dom}(f)) \rightarrow \mathbb{R}$ with

$$
S_{(f, g)}(x, y)=\left\{\begin{array}{l}
f^{-}(y)+\int_{y}^{x} g^{-}(u) d u \quad x \geqslant y \\
f^{-}(y)-\int_{x}^{y} g^{+}(u) d u \quad x<y .
\end{array}\right.
$$

In other words, $s(f, g)$ is the upper envelop of the one parameter family of functions $x \mapsto S_{(f, g)}(x, y)$. We will now construct $s$ explicitly, which gives a constructive proof of the lemma. Consider the common refinement $\mathscr{A}=\left\{c_{0}, c_{1}, c_{2}, \cdots, c_{n}\right\}$, with $v=c_{0}<c_{1}<c_{2}$ $<\cdots<c_{n}=w$, of the two partitions of $O$ by the associated intervals of $f$ and $g$ as described in Section 2. In the open intervals $\left(c_{i-1}, c_{i}\right)(i=1, \cdots, n)$, the restrictions 
$f^{-} \uparrow_{\left(c_{i-1}, c_{i}\right)}=p_{i}^{-}$and $f^{+} \uparrow_{\left(c_{i-1}, c_{i}\right)}=p_{i}^{+}$are polynomials. Let $q^{-}, q^{+}:[v, w] \rightarrow \mathbb{R}$ be the continuous piecewise polynomial functions with, say, $q^{-}(v)=q^{+}(v)=0$ such that for all $x \in O \backslash \mathscr{A}$ we have $\left(q^{-}\right)^{\prime}(x)=g^{-}(x)$ and $\left(q^{+}\right)^{\prime}(x)=g^{+}(x)$. Let $q_{i}^{-}$and $q_{i}^{+}$be, respectively, the restrictions of $q^{-}$and $q^{+}$to $\left[c_{i-1}, c_{i}\right]$. Then, for $y \in\left(c_{i-1}, c_{i}\right)$ we have $S_{(f, g)}(x, y)=p_{i}^{-}(y)+Q_{i}(x, y)$ with

$$
Q_{i}(x, y)= \begin{cases}q^{-}(x)-q_{i}^{-}(y) & x \geqslant y \\ q^{+}(x)-q_{i}{ }^{+}(y) & x<y\end{cases}
$$

where $Q_{i}:[v, w] \times\left[c_{i-1}, c_{i}\right] \rightarrow \mathbb{R}$ is a continuous piecewise polynomial function. For fixed $x \in[v, w]$, the left and right partial derivatives of $Q_{i}$ with respect to $y \in\left(c_{i-1}, c_{i}\right)$ exist and are given by

$$
\frac{\partial^{-} Q_{i}(x, y)}{\partial y}=-\left(q_{i}^{+}\right)^{\prime}(y) \quad \frac{\partial^{+} Q_{i}(x, y)}{\partial y}=-\left(q_{i}^{-}\right)^{\prime}(y) .
$$

The upper envelop can be expressed as

$$
s(f, g)(x)=\sup _{1 \leqslant i \leqslant n}\left(\sup _{y \in\left[c_{i-1}, c_{i}\right]}\left(p_{i}^{-}(y)+Q_{i}(x, y)\right)\right) .
$$

The graph of the upper envelop for $y \in\left[c_{i-1}, c_{i}\right]$, that is, the graph of

$$
x \mapsto \sup _{y \in\left[c_{i-1}, c_{i}\right]} p_{i}^{-}(y)+Q_{i}(x, y),
$$

contains a finite number of segments each of which can be one of two types:

- A segment of the graph of $p_{i}^{-}$in an interval $[\alpha, \beta] \subseteq\left[c_{i-1}, c_{i}\right]$, where, for $y \in[\alpha, \beta]$, the graph of $x \mapsto Q_{i}(x, y)$ is, in a neighbourhood of $y$, below the tangent to $p_{i}^{-}$at $y$. For this to happen, we must have the following two necessary conditions:

- $\alpha=c_{i-1}$ or $\left(p_{i}^{-}\right)^{\prime}(\alpha)=\left(q_{i}^{+}\right)^{\prime}(\alpha)$ or $\left(p_{i}^{-}\right)^{\prime}(\alpha)=\left(q_{i}^{-}\right)^{\prime}(\alpha)$.

- $\beta=c_{i}$ or $\left(p_{i}^{-}\right)^{\prime}(\beta)=\left(q_{i}^{+}\right)^{\prime}(\beta)$ or $\left(p_{i}^{-}\right)^{\prime}(\beta)=\left(q_{i}^{-}\right)^{\prime}(\beta)$.

- A segment of the graph of $x \mapsto p_{i}^{-}(y)+Q_{i}(x, y)$ for some $y \in\left[c_{i-1}, c_{i}\right]$, where either $y=c_{i-1}$ or $y=c_{i}$ or $\left(p_{i}^{-}\right)^{\prime}(y)=\left(q_{i}^{+}\right)^{\prime}(y)$ or $\left(p^{-} i\right)^{\prime}(y)=\left(q_{i}^{-}\right)^{\prime}(y)$.

Let $K_{i}=\left\{c_{i-1}, c_{i}\right\} \cup\left\{y \in\left(c_{i-1}, c_{i}\right) \mid\left(p_{i}^{-}\right)^{\prime}(y)=\left(q_{i}^{+}\right)^{\prime}(y)\right.$ or $\left.\left(p_{i}^{-}\right)^{\prime}(y)=\left(q_{i}^{-}\right)^{\prime}(y)\right\}$. Since $p_{i}^{-}, q_{i}^{-}$ and $q_{i}^{+}$are semi-rational polynomials, $K_{i}$ is, for each $i=1, \cdots, n$, a finite set of algebraic numbers and we have

$$
\sup _{y \in\left[c_{i-1}, c_{i}\right]} p_{i}^{-}(y)+Q_{i}(x, y)=\sup _{y \in K_{i}} p_{i}^{-}(y)+Q_{i}(x, y) .
$$

Recalling the definition of $Q_{i}$, we see that for each $y \in K_{i}$, we have

$$
p_{i}^{-}(y)+Q_{i}(x, y)= \begin{cases}q^{-}(x)+\gamma^{-} & x \geqslant y \\ q^{+}(x)+\gamma^{+} & x<y,\end{cases}
$$

where $\gamma^{-}=p_{i}^{-}(y)-q_{i}^{-}(y)$ and $\gamma^{+}=p_{i}^{-}(y)-q_{i}^{+}(y)$ are algebraic numbers. Recall that the comparison of algebraic numbers is decidable. It follows that the supremum in Equation 5 consists of a continuous piecewise polynomial function such that each polynomial defining the function is a rational polynomial translated by an algebraic number and defined on an interval with algebraic endpoints. 
Now put $K=\bigcup_{1 \leqslant i \leqslant n} K_{i}=\left\{d_{j} \mid 0 \leqslant j \leqslant m\right\}$ with $v=d_{0}<d_{1}<\cdots<d_{m}=w$. Then, there are rational polynomials $r_{j}$ and algebraic numbers $\gamma_{j}(1 \leqslant j \leqslant m)$ such that $s(f, g)(x)=r_{j}(x)+\gamma_{j}$ for $x \in\left[d_{j-1}, d_{j}\right]$, as required. Similarly, it follows that $t(f, g)$ is a piecewise semi-rational polynomial in $O$.

Theorem 6.8. The relation Cons $\subseteq D^{0}[0,1] \times D^{0}[0,1]$ is decidable on the basis of semirational polynomial step functions.

Proof. Let $f, g \in D^{0}[0,1]$ be semi-rational polynomial step functions. We note that $(f, g) \in$ Cons iff $(f, g)$ is consistent in each connected open component of $\operatorname{dom}(g)$ with nonempty intersection with $\operatorname{dom}(f)$. Fix a connected component $O$ of the domain of $g$. Then, using the notation in Lemma 6.7, $(f, g)$ will be consistent in $O$ iff $\forall x \in O . s(f, g)(x) \leqslant f^{+}(x)$. Since $s(f, g)$ and $f^{+}$are piecewise semi-rational polynomials, it follows by Proposition 6.6, that the relation $\forall x \in O . s(f, g)(x) \leqslant f^{+}(x)$ is decidable, and hence the relation Cons is also.

Definition 6.9. The domain of $C^{1}$ functions on $[0,1]$ is the subdomain of $\left(D^{0}[0,1]\right)^{2}$ given by

$$
D^{1}[0,1]=\left\{(f, g) \in\left(D^{0}[0,1]\right)^{2} \mid(f, g) \in \text { Cons }\right\} .
$$

Corollary 6.10. $D^{1}[0,1]$ is an $\omega$-continuous bounded complete depo that can be given an effective structure.

Proof. We just note that $D^{1}[0,1]$ is a Scott-closed subset of the $\omega$-continuous bounded complete dcpo $(\mathbf{I}[0,1] \rightarrow \mathbf{I R})^{2}$ and is therefore itself an $\omega$-continuous bounded complete dcpo. Because Cons is decidable on the basis of step functions (and also on the polynomial step functions), $D^{1}[0,1]$ can be provided with an effective structure. More specifically, we start with a standard effective structure on $D^{0}[0,1]$ : a standard enumeration of the basis of rational step functions, with decidable predicates for $\sqsubseteq_{D^{0}}, \ll_{D^{0}}, \operatorname{Con}_{\left(D^{0}, \sqsubseteq\right)}$ and $\operatorname{Con}_{\left(D^{0}, \ll\right)}$ when restricted to the basis - see Smyth (1977) and Plotkin (1981). We take the product of the effectively given $D^{0}[0,1]$ with itself, together with the decidable Cons on the pairs of basis elements. Since the basic relations in $D^{1}[0,1]$ are obtained componentwise from that of $\left(D^{0}[0,1]\right) \times\left(D^{0}[0,1]\right)$, we will obtain an enumeration of the induced basis of $D^{1}[0,1]$ with decidable predicates for $\sqsubseteq_{D^{1}}$, $\ll_{D^{1}}, \operatorname{Con}_{\left(D^{1}, \sqsubseteq\right)}$ and $\operatorname{Con}_{\left(D^{1}, \ll\right)}$ when restricted to this basis.

Proposition 6.11. Let $(f, g) \in D^{1}$ such that $f$ and $g$ preserve maximal elements in some open set $O \subseteq \operatorname{dom}(f) \cap \operatorname{dom}(g)$. Then for all $x \in O$, the induced maps $f, g: O \rightarrow \mathbb{R}$ satisfy $f^{\prime}(x)=g(x)$ for all $x \in O$.

Let the derivative operator

$$
\frac{D}{D x}: D^{1}[0,1] \rightarrow D^{0}[0,1]
$$

be the projection to the second component, that is, $\frac{D}{D x}(f, g)=g$. Then $\frac{D}{D x}$ is clearly continuous and we have the following result. 


\section{Proposition 6.12.}

$$
\frac{D}{D x}(f, g) \sqsubseteq \prod\left\{\frac{d}{d x} \mathbf{I} h \mid \mathbf{I} h \in \uparrow f \cap \int g\right\} .
$$

Proof. If $\mathbf{I} h \in \uparrow f \cap \int g$, then $g \sqsubseteq \frac{d}{d x} \mathbf{I} h$ by Corollary 4.5 .

Let $\Gamma^{1}: C^{0}[0,1] \rightarrow D^{1}[0,1]$ be defined by $\Gamma^{1}(f)=\left(\mathbf{I} f, \frac{d \mathbf{I} f}{d x}\right)$.

Theorem 6.13. The map $\Gamma^{1}$ is an embedding into the set of maximal elements of $D^{1}$.

Proof. We first check that $\Gamma^{1}$ is well-defined. By Corollary 4.5, we have $\mathbf{I} f \in \int \frac{d \mathbf{I} f}{d x}$ and thus $\left(\mathbf{I} f, \frac{d \mathbf{I} f}{d x}\right) \in D^{1}$. We now show that $\left(\mathbf{I} f, \frac{d \mathbf{I} f}{d x}\right)$ is maximal. If $\frac{d \mathbf{I} f}{d x} \sqsubseteq g$ and $(\mathbf{I} f, g) \in D^{1}$, then for any $a \searrow b \sqsubseteq g$ we have $\mathbf{I} f \in \int g \subseteq \delta(a, b)$, which implies $a \searrow b \sqsubseteq \frac{d \mathbf{I} f}{d x}$. Thus $g \sqsubseteq \frac{d \mathbf{I} f}{d x}$, that is, $g=\frac{d \mathbf{I} f}{d x}$ and $\left(f, \frac{d \mathbf{I} f}{d x}\right)$ is maximal. That $\Gamma^{1}$ is an embedding follows from Theorem 2.2.

The map $\Gamma^{1}$ restricts to give an embedding of locally Lipschitz functions into the set of maximal elements of $D^{1}$. Note that $f \in C^{0}[0,1]$ is locally Lipschitz iff $\frac{d \mathbf{I} f}{d x}(y) \neq \perp$ for all $y \in \mathbf{I}[0,1]$. Also, $\Gamma^{1}$ restricts to give an embedding of piecewise $C^{1}$ functions into the set of maximal elements of $D^{1}$. It is easy to see that a function $f \in C^{0}[0,1]$ is piecewise $C^{1}$ iff $\frac{d \mathbf{I} f}{d x}(\{y\})$ is non-maximal for all but a finite number of $y \in[0,1]$. Moreover, if we consider $C^{1}$ functions with their $C^{1}$ norm we obtain the following corollary.

Corollary 6.14. The map $\Gamma^{1}$ restricts to give a topological embedding $\Gamma^{1}: C^{1}[0,1] \rightarrow$ $D^{1}[0,1]$, which makes the following diagram commute:

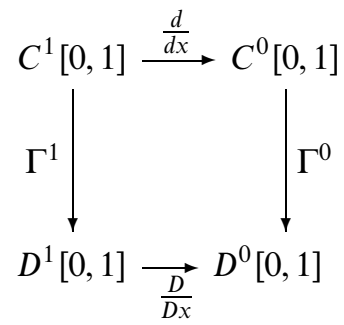

Finally, note that, by analogy, we can define

$$
D_{\mathrm{r}}^{1}=\left\{(f, g) \in D_{\mathrm{r}}^{0} \times D_{\mathrm{r}}^{0} \mid(f, g) \in \uparrow f \cap \int_{\mathrm{r}} g \neq \varnothing\right\},
$$

and all the results in this section will have their analogues for $D_{\mathrm{r}}^{1}$.

\section{A domain-theoretic version of Picard's theorem}

Consider a vector field $v: O \rightarrow \mathbb{R}$ for some open subset $O \subseteq \mathbb{R}^{2}$ and a point $\left(t_{0}, x_{0}\right) \in O$. The following theorem, whose proof is based on the Banach fix-point theorem, is referred to as Picard's theorem and is at the foundation of the theory of differential equations.

Theorem 7.1. (Kolmogorov and Fomin 1975) If $v$ is continuous in $O$ and uniformly Lipschitz in the second argument, then the initial value problem $\dot{x}=v(t, x)$ with $x\left(t_{0}\right)=x_{0}$ has a unique solution in a neighbourhood of $t_{0}$ and can be obtained as the unique fix-point 
of the operator

$$
P: f \mapsto \lambda t .\left(x_{0}+\int_{t_{0}}^{t} v(t, f(t)) d t\right): C^{0}\left[t_{0}-\delta, t_{0}+\delta\right] \rightarrow C^{0}\left[t_{0}-\delta, t_{0}+\delta\right]
$$

in some small enough neighbourhood $\left|t-t_{0}\right| \leqslant \delta$.

Picard's theorem can be easily extended to vector fields $v: O \rightarrow \mathbb{R}^{n}$, with $O \subseteq \mathbb{R}^{n+1}$, which gives a basis for solving systems of ordinary differential equations. In this section we present a domain-theoretic version of this theorem for $n=1$, which, like Picard's theorem, can easily be extended to any dimension $n \geqslant 1$. The result is more general than the classical theorem, as it allows for the initial condition to be given by two approximations, one to the solution itself and one to the derivative of the solution at the initial time. If only the classical initial value problem is considered, a simpler domain-theoretic extension of the Picard's theorem can be obtained as presented in the follow-up paper Edalat and Pattinson (2003).

The classical operator $P$ above can be reformulated as the composition of two operators $U, A_{v}:\left(C^{0}\left[t_{0}-\delta, t_{0}+\delta\right]\right)^{2} \rightarrow\left(C^{0}\left[t_{0}-\delta, t_{0}+\delta\right]\right)^{2}$ on pairs $(f, g)$, where $f$ gives an approximation to the solution and $g$ gives an approximation to the derivative of the solution. The map $A_{v}$ upgrades the information on the derivative and $U$ upgrades the information on the function itself:

$$
U(f, g)=\left(\lambda t .\left(x_{0}+\int_{t_{0}}^{t} g d t\right), g\right) \quad A_{v}(f, g)=(f, \lambda t . v(t, f(t)) .
$$

We have $P(f)=\pi_{0}\left(U \circ A_{v}(f, g)\right)$, for any $g$, where $\pi_{0}$ is projection to the first component. The unique fix-point $(f, g)$ of $U \circ A_{v}$ will satisfy $f^{\prime}=g=\lambda t \cdot v(t, f(t))$.

We consider a domain-theoretic framework for differential equations, and define similar operators in that setting. We define

$$
H:(\mathbf{I}[0,1] \times \mathbf{I} \mathbb{R} \rightarrow \mathbf{I} \mathbb{R}) \times D^{0} \rightarrow D^{0}
$$

by

$$
H(v, f): t \mapsto v(t, f(t))
$$

In analogy with the classical vector field above, we consider $v \in(\mathbf{I}[0,1] \times \mathbf{I R} \rightarrow \mathbf{I R})$ as an interval-valued vector field with interval input. The map $H$ is Scott continuous and we put $H_{v}: D^{0} \rightarrow D^{0}$ with $H_{v}(f)=H(v, f)$. Let

$$
\text { Ap }:(\mathbf{I}[0,1] \times \mathbf{I} \mathbb{R} \rightarrow \mathbf{I} \mathbb{R}) \times\left(D^{0} \times D^{0}\right) \rightarrow D^{0} \times D^{0}
$$

with $\operatorname{Ap}(v,(f, g))=(f, H(v, f))$, and let $\operatorname{Ap}_{v}: D^{0} \times D^{0} \rightarrow D^{0} \times D^{0}$ with $\operatorname{Ap}_{v}(f, g)=$ $\operatorname{Ap}(v,(f, g))$. Also define

$$
\text { Up : } D^{1} \rightarrow D^{1}
$$

with $U p(f, g)=(\mathbf{I}[s(f, g), t(f, g)], g)$ and put $\cup_{1}(f, g)=\mathbf{I}[s(f, g), t(f, g)]$.

\section{Proposition 7.2.}

(i) The maps $A p_{v}$ and $U p$ are continuous.

(ii) $U p_{1}(f, g) \sqsupseteq\left(\bigsqcup\left\{h \mid \uparrow h \cap \int g=\uparrow f \cap \int g\right\}\right)$.

(iii) $U \mathrm{p}_{1}\left(\mathrm{Up}_{1}(f, g), g\right)=U \mathrm{p}_{1}(f, g)$. 


\section{Proof.}

(i) The continuity of Ap follows from the continuity of $H$, and the continuity of Up from the continuity of $s$ and $t$ (Proposition 6.3).

(ii) Note that for $k_{1}, k_{2} \in C^{0}$, with $k_{1} \leqslant k_{2}$, we have $\mathbf{I}\left[k_{1}, k_{2}\right]=\mathbf{I} k_{1} \sqcap \mathbf{I} k_{2}$. Let $h \in D^{0}$ satisfy $\uparrow h \cap \int g=\uparrow f \cap \int g$. Since $\mathbf{I} s(f, g), \mathbf{I} t(f, g) \sqsupseteq h$, we get

$$
\mathrm{Up}_{1}(f, g)=\mathbf{I}[s(f, g), t(f, g)]=(\mathbf{I} s(f, g)) \sqcap(\mathbf{I} t(f, g)) \sqsupseteq h .
$$

(iii) Recall that, for each component $O$ of $\operatorname{dom}(g)$ that intersects $\operatorname{dom}(f)$, the map $s(f, g)$ : $O \rightarrow \mathbb{R}$ is the least function $h \in C^{0}(O)$ with $h \geqslant f^{-}$and $\mathbf{I} h \in \int g$; the dual property holds for $t(f, g)$. Since $U_{p_{1}}(f, g)=\mathbf{I}[s(f, g), t(s, f)] \in \int g$, we get $s\left(U_{p_{1}}(f, g), g\right)=s(f, g)$ and $t\left(\mathrm{Up}_{1}(f, g), g\right)=t(f, g)$.

Unlike Up, the map $\operatorname{Ap}_{v}$ does not take $D^{1}[0,1]$ to itself. We need to work in a subdomain of $D^{1}[0,1]$, dependent on $v$, that is preserved by $\mathrm{Ap}_{v}$. In order to define such a subdomain, we first define a stronger version of consistency. We say that $(f, g) \in D^{1}$ is strongly consistent, and write $(f, g) \in$ SCons, if $(f, h) \in$ Cons for all $h \sqsupseteq g$.

Proposition 7.3. If $(f, g) \in$ SCons, then:

(i) $(f, h) \in$ SCons for all $h \sqsupseteq g$.

(ii) $\cup \mathrm{p}(f, g) \in$ SCons.

Proof. (i) is trivial. To see (ii), let $h \sqsupseteq g$. Since $(f, g) \in$ SCons, we have $(f, h) \in$ Cons. But then $\operatorname{Up}(f, h)=\left(\mathrm{Up}_{1}(f, h), h\right) \in$ Cons. Now, by the monotonicity of $\mathrm{Up}_{1}$, we have $\mathrm{Up}_{1}(f, g) \sqsubseteq \mathrm{Up}_{1}(f, h)$, and therefore $\left(\mathrm{Up}_{1}(f, g), h\right) \in$ Cons. This shows that $\mathrm{Up}(f, g)=$ $\left(\mathrm{Up}_{1}(f, g), g\right) \in$ SCons.

Proposition 7.4. Strong consistency is closed under taking lubs of increasing chains, that is, if $\left(f_{i}, g_{i}\right) \in$ SCons where $\left(f_{i}\right)$ and $\left(g_{i}\right)$ are increasing chains in $D^{1}$, then

$$
\left(\bigsqcup f_{i}, \bigsqcup g_{i}\right) \in \text { SCons }
$$

Proof. Suppose $\left(f_{i}, g_{i}\right) \in$ SCons where $\left(f_{i}\right)_{i \in \omega}$ and $\left(g_{i}\right)_{i \in \omega}$ are increasing chains in $D^{1}$. We want to show that $\left(\bigsqcup_{i \in \omega} f_{i}, \bigsqcup_{i \in \omega} g_{i}\right) \in$ SCons. Let $\bigsqcup_{i \in \omega} g_{i} \sqsubseteq g$. Then $g_{i} \sqsubseteq g$ for all $i \geqslant 0$. Hence, by strong consistency of $\left(f_{i}, g_{i}\right)$, we have $\left(f_{i}, g\right) \in$ Cons. Since Cons is Scott closed, we get $\left(\bigsqcup_{i \in \omega} f_{i}, g\right) \in$ Cons.

We now define a subdomain of $D^{1}[0,1]$ that is preserved by the updating maps. Let $D_{v}^{1}=\left\{(f, g) \in D^{1} \mid(f, g) \in\right.$ SCons \& $\left.g \sqsubseteq H(v, f)\right\}$.

Proposition 7.5. $D_{v}^{1}$ is a depo and the two maps $\mathrm{Ap}_{v}$ and $U \mathrm{p}$ take $D_{v}^{1}$ into itself.

Proof. By Proposition 7.4, SCons is closed under the lubs of increasing chains. On the other hand, suppose $\left(\left(f_{i}, g_{i}\right)\right)_{i \in \omega}$ is an increasing chain with $g_{i} \sqsubseteq H\left(v, f_{i}\right)$ for $i \in \omega$. Then by continuity of $H_{v}$, we get $\bigsqcup_{i \in \omega} g_{i} \sqsubseteq \bigsqcup_{i \in \omega} H\left(v, f_{i}\right)=\bigsqcup_{i \in \omega} H_{v}\left(f_{i}\right)=H_{v}\left(\bigsqcup_{i \in \omega} f_{i}\right)$.

Let $(f, g) \in D_{v}^{1}$. Then $\operatorname{Ap}_{v}(f, g)=(f, H(v, f))$. Since $g \sqsubseteq H(v, f)$ and $(f, g) \in$ SCons, by Proposition 7.3(i), we get the strong consistency of $(f, H(v, f))$. It follows that $\operatorname{Ap}_{v}(f, g) \in D_{v}^{1}$, as the second condition is trivially satisfied. On the other hand, 
$\mathrm{Up}(f, g)=\left(\mathrm{Up}_{1}(f, g), g\right) \in$ SCons by Proposition 7.3(ii). Finally, by monotonicity of $H_{v}$, we get $g \sqsubseteq H_{v}(f) \sqsubseteq H_{v}(\cup \mathrm{p}(f, g))$. Hence, $\cup \mathrm{p}(f, g) \in D_{v}^{1}$.

We now have a domain-theoretic generalisation of Picard's theorem. Let $P_{v}: D_{v}^{1} \rightarrow D_{v}^{1}$ be given by $P_{v}=U \mathrm{p} \circ \operatorname{Ap}_{v}$. For $\left(f_{0}, g_{0}\right) \in D_{v}^{1}$, let $D_{v,\left(f_{0}, g_{0}\right)}^{1}=\uparrow\left(f_{0}, g_{0}\right) \cap D_{v}^{1}$. Then $D_{v,\left(f_{0}, g_{0}\right)}^{1}$ is a dcpo with least element $\left(f_{0}, g_{0}\right)$. Thus we obtain the following theorem.

Theorem 7.6. The restriction $P_{v,\left(f_{0}, g_{0}\right)}=P_{v} \uparrow_{D_{v,\left(f_{0}, g_{0}\right)}^{1}}: D_{v,\left(f_{0}, g_{0}\right)}^{1} \rightarrow D_{v,\left(f_{0}, g_{0}\right)}^{1}$ has a least fix-point $\left(f_{s}, g_{s}\right)$ with $f_{0} \sqsubseteq f_{s}$ and $g_{0} \sqsubseteq g_{s}$.

\subsection{Width of the fix-point $f_{s}$}

We first obtain a bound for the update $\operatorname{Up}(f, g)$.

Proposition 7.7. We have $h \in \int g$ iff, for all $x, x_{1}, x_{2} \in \mathbf{I}[0,1]$ with $x \sqsubseteq x_{1}, x_{2}$, we have $\left(x_{2}-x_{1}\right) g(x) \sqsubseteq h\left(x_{2}\right)-h\left(x_{1}\right)$.

Proof. For the 'Only if part', let $a \searrow b \sqsubseteq g$. Then a simple calculation shows that for all $x_{1}, x_{2} \sqsupseteq x$ we have

$$
\left(x_{2}-x_{1}\right)(a \searrow b)(x) \sqsubseteq\left(x_{2}-x_{1}\right) b \sqsubseteq h\left(x_{2}\right)-h\left(x_{1}\right) .
$$

Taking the supremum over all step functions way-below $g$, the result follows by the Scott continuity of the interval product $-\times-:(u, v) \mapsto u v: \mathbf{I R} \times \mathbf{I} \mathbb{R} \rightarrow \mathbf{I} \mathbb{R}$.

For the 'If part', let $a \searrow b \sqsubseteq g$. It suffices to show that $h \in \delta(a, b)$. Suppose $x_{1}, x_{2} \gg a$ and put $x=x_{1} \sqcap x_{2}$. Then $x \gg a$ and $x_{1}, x_{2} \sqsupseteq x$. Thus,

$$
\left(x_{2}-x_{1}\right) b=\left(x_{2}-x_{1}\right)(a \searrow b)(x) \sqsubseteq\left(x_{2}-x_{1}\right) g(x) \sqsubseteq h\left(x_{2}\right)-h\left(x_{1}\right),
$$

and hence, $h \in \delta(a, b)$.

Corollary 7.8. I $h \in \int g$ iff, for all $x, y \in \operatorname{dom}(g)$ with $x \leqslant y$, we have

$$
(y-x) \underline{g([x, y])} \leqslant f(y)-f(x) \leqslant(y-x) \overline{g([x, y])} .
$$

Assume now that $h \in \uparrow f \cap \int g$. For all $x, x_{1}, x_{2} \in \mathbf{I}[0,1]$, with $x \sqsubseteq x_{1}, x_{2}$, from Proposition 7.7, we get

$$
f\left(x_{1}\right)+\left(x_{2}-x_{1}\right) g(x) \sqsubseteq h\left(x_{1}\right)+\left(x_{2}-x_{1}\right) g(x) \sqsubseteq h\left(x_{2}\right)-h\left(x_{1}\right)+h\left(x_{1}\right) \sqsubseteq h\left(x_{2}\right)
$$

and, therefore

$$
\bigsqcup\left\{f\left(x_{1}\right)+\left(x_{2}-x_{1}\right) g(x) \mid x \sqsubseteq x_{1} \& x \sqsupseteq x_{2}\right\} \sqsubseteq h\left(x_{2}\right) .
$$

By Proposition 7.2(ii), it follows that

$$
\mathrm{Up}(f, g)\left(x_{2}\right) \sqsupseteq \bigsqcup\left\{f\left(x_{1}\right)+\left(x_{2}-x_{1}\right) g(x) \mid x \sqsubseteq x_{1} \& x \sqsupseteq x_{2}\right\} .
$$

Let $\left(f_{s}, g_{s}\right)$ be the fix-point of Theorem 7.6, so that $\operatorname{Up}\left(f_{s}, g_{s}\right)=f_{s}$. Hence, by Equation 8, we have

$$
f_{s}\left(x_{2}\right) \sqsupseteq \bigsqcup\left\{f_{s}\left(x_{1}\right)+\left(x_{2}-x_{1}\right) g_{s}(x) \mid x \sqsubseteq x_{1} \& x \sqsupseteq x_{2}\right\} .
$$


In particular, for real numbers $t_{1}$ and $t_{2}$, with $t_{1}<t_{2}$, considered as maximal elements of $\mathbf{I}[0,1]$,

$$
f_{s}\left(t_{2}\right) \sqsupseteq f_{s}\left(t_{1}\right)+\left(t_{2}-t_{1}\right) g_{s}\left(\left[t_{1}, t_{2}\right]\right),
$$

where for convenience we have written $t_{i}$ for $\left\{t_{i}\right\}$.

We denote the width of an interval $a$ by $w(a)=\bar{a}-\underline{a}$. For $f \in D^{0}[0,1]$, let $w_{f}:[0,1] \rightarrow \mathbb{R}$ be given by $w_{f}(x)=w(f(\{x\})$. From Equation 9 we get

$$
w_{f_{s}}\left(t_{2}\right) \leqslant w_{f_{s}}\left(t_{1}\right)+\left(t_{2}-t_{1}\right) w_{g_{s}}\left(\left[t_{1}, t_{2}\right]\right) .
$$

Let $S$ be the set of all finite increasing sequences

$$
p=\left\{t_{0}, t_{1}, t_{2}, \cdots, t_{n} \mid t_{0}<t_{1}<t_{2}<. .<t_{n}=t\right\} .
$$

We have, from Equation 10,

$$
w_{f_{s}}(t) \leqslant w_{f_{s}}\left(t_{0}\right)+\sum_{i=1, n} w_{g_{s}}\left(\left[t_{i-1}, t_{i}\right]\right)\left(t_{i}-t_{i-1}\right) .
$$

For a given sequence $p=\left\{t_{0}, t_{1}, t_{2}, \ldots, t_{n} \mid t_{0}<t_{1}<t_{2}<. .<t_{n}=t\right\}$, we use $\Theta_{p}$ to denote the classical step function defined, for $u \in\left[t_{0}, t\right]$, by $\Theta_{p}(u)=w_{g_{s}}\left(\left[t_{i-1}, t_{i}\right]\right)$ if $u \in\left(t_{i-1}, t_{i}\right)$ and $\Theta_{p}\left(t_{i}\right)=\max \left(w_{g_{s}}\left(\left[t_{i-1}, t_{i}\right]\right), w_{g_{s}}\left(\left[t_{i}, t_{i+1}\right]\right)\right)$. Equation 11 can be written as

$$
w_{f_{s}}(t) \leqslant w_{f_{s}}\left(t_{0}\right)+\int_{t_{0}}^{t} \Theta_{p}(u) d u .
$$

Consider the family of sequences $\left(p_{k}\right)_{k \in \omega}$ where

$$
p_{k}=\left\{t_{0}, t_{0}+2^{-k}\left[t-t_{0}\right], t_{0}+2 \cdot 2^{-k}\left[t-t_{0}\right], t_{0}+3 \cdot 2^{-k}\left[t-t_{0}\right], \ldots, t_{0}+2^{k} \cdot 2^{-k}\left[t-t_{0}\right]\right\} .
$$

The sequence $\Theta_{p_{k}}$ is decreasing and converges pointwise to $w_{g_{s}}$, which is upper semicontinuous. Hence, by the monotone convergence theorem, we have the following proposition from Equation 12.

\section{Proposition 7.9.}

$$
w_{f_{s}}(t) \leqslant w_{f_{s}}\left(t_{0}\right)+\int_{t_{0}}^{t} w_{g_{s}}(u) d u .
$$

\subsection{The Lipschitz case}

Let $\left(f_{s}, g_{s}\right)$ be the fix-point of the theorem. We have

$$
g_{s}(u)=v\left(u, f_{s}(u)\right) \text {. }
$$

We assume now that $v: \mathbf{I}[0,1] \times \mathbf{I} \mathbb{R} \rightarrow \mathbf{I} \mathbb{R}$ satisfies a Lipschitz-like condition with constant $K$ with respect to its second argument uniformly in the maximal elements of its first argument; in other words, for any maximal element (that is, real) $t$, we assume $w(v(t, X)) \leqslant K w(X)$. Equation 13 then gives $w\left(g_{s}(u)\right) \leqslant K w\left(f_{s}(u)\right)$, which, together with Proposition 7.9 , leads to

$$
w_{f_{s}}(t) \leqslant w_{f_{s}}\left(t_{0}\right)+K \int_{t_{0}}^{t} w_{f_{s}}(u) d u .
$$


A simple inductive proof shows that for each integer $n \geqslant 0$

$$
\begin{aligned}
w_{f_{s}}(t) \leqslant & w_{f_{s}}\left(t_{0}\right)\left(1+K\left(t-t_{0}\right)+\cdots+K^{n}\left(t-t_{0}\right)^{n} / n !\right. \\
& \left.+K^{n} \int_{t_{0}}^{t} \int_{t_{0}}^{u_{0}} \cdots \int_{t_{n}}^{u_{n}} w_{f_{s}}\left(u_{n}\right) d u_{0} d u_{1} \cdots d u_{n}\right) .
\end{aligned}
$$

Since $f_{s}$ is bounded, we have a bound $B$ on $w_{f_{s}}\left(u_{n}\right)$ and the remainder is bounded by

$$
\begin{aligned}
K^{n} \int_{t_{0}}^{t} \int_{t_{0}}^{u_{0}} \cdots \int_{t_{n}}^{u_{n}} w_{f_{s}}\left(u_{n}\right) d u_{0} d u_{1} \cdots d u_{n} & \leqslant K^{n} B \int_{t_{0}}^{t} \int_{t_{0}}^{u_{0}} \cdots \int_{t_{n}}^{u_{n}} d u_{0} d u_{1} \cdots d u_{n} \\
& =K^{n}\left(t-t_{0}\right)^{(n+1)} /(n+1) !
\end{aligned}
$$

This remainder goes to 0 when $n \rightarrow \infty$ for any fixed $t$. By taking the limit, we obtain $w_{f_{s}}(t) \leqslant w_{f_{s}}\left(t_{0}\right) e^{K\left(t-t_{0}\right)}$. Since, the same holds for $t<t_{0}$, we conclude with the following result.

Proposition 7.10.

$$
w_{f_{s}}(t) \leqslant w_{f_{s}}\left(t_{0}\right) e^{K\left|t-t_{0}\right|}
$$

\subsection{Picard's theorem revisited}

Proposition 7.11. If $\left(T_{i} \searrow X_{i}\right)_{i \in I}$ is a family of consistent step functions, then

$$
\left(\bigsqcup_{i \in I}\left(T_{i} \searrow X_{i}\right),\left(\prod_{i \in I} T_{i}\right) \searrow B\right)
$$

is a strongly consistent pair iff $X_{i}-X_{j} \sqsubseteq\left(T_{i}-T_{j}\right) B$ for all $i, j \in I$.

Proof. First note that for $i, j \in I$ we have $X_{i}-X_{j} \sqsubseteq\left(T_{i}-T_{j}\right) B$ iff, for each $u \in B$, there exists a straight line with slope $u$ that intersects the four vertical sides of the rectangles $\left(T_{i}, X_{i}\right)$ and $\left(T_{j}, X_{j}\right)$ when $i \neq j$, or the two vertical sides of $\left(T_{i}, X_{i}\right)$ when $i=j$. The 'Only if part' then follows immediately.

For the 'If' part, fix $u \in B$. For each unordered pair $i, j \in I$, consider the family $\mathscr{L}_{i j}$ of parallel straight lines with slope $u$, which intersect the four vertical sides of the rectangles $\left(T_{i}, X_{i}\right)$ and $\left(T_{j}, X_{j}\right)$ when $i \neq j$, or the two vertical sides of $\left(T_{i}, X_{i}\right)$ when $i=j$. For a given unordered pair $i, j \in I$, let $l_{i j}$ and $g_{i j}$ be the infimum and the supremum, respectively, of the non-empty set $\left\{L(0) \mid L \in \mathscr{L}_{i j}\right\}$. By assumption and the above observation, the set of intervals $\left\{\left[l_{i j}, g_{i j}\right] \mid i, j \in I\right\}$ have pairwise non-empty intersections. Hence, $I=\bigcap_{i, j \in I}\left[l_{i j}, g_{i j}\right] \neq \varnothing$ and any line with slope $u$ passing through any point of $I$ intersects the two vertical edges of $\left(T_{i}, X_{i}\right)$ for all $i \in I$.

Theorem 7.12. Let $v: O \rightarrow \mathbb{R}$ be continuous in the open set $O \subseteq \mathbb{R}^{2}$ and $\left(t_{0}, x_{0}\right) \in O$. If $v$ is Lipschitz in its second argument uniformly in the first argument, then the solution of the initial value problem $\dot{x}=v(t, x)$ with $x\left(t_{0}\right)=x_{0}$ given by the classical Picard's theorem coincides with the domain-theoretic solution. 
Proof. Let $A \subset O$ be a compact rectangle with $\left(t_{0}, x_{0}\right) \in A^{\circ}$ such that, for some $M>0$, we have $|v(t, x)| \leqslant M$ for all $(t, x) \in A$. Let $\left(T_{i}, X_{i}\right)_{i \in \omega}$ be any shrinking nested sequence of compact rectangles such that:

$-\left(T_{i}, X_{i}\right) \subset A^{\circ}$ all $i \in \omega$;

- $\left(t_{0}, x_{0}\right)$ is the centre of $\left(T_{i}, X_{i}\right)$ for all $i \in \omega$;

- $w\left(X_{i}\right)=M w\left(T_{i}\right)$ for all $i \in \omega$; and

$-\bigsqcup_{i}\left(T_{i}, X_{i}\right)=\left(\left\{t_{0}\right\},\left\{x_{0}\right\}\right)$.

We now consider the domain-theoretic Picard's theorem for the canonical extension $\mathbf{I} v: \mathbf{I} T_{0} \times \mathbf{I R} \rightarrow \mathbf{I R}$. Let $B=[-M, M]$. Then $v$ satisfies $A \searrow B \sqsubseteq \mathbf{I} v$. By Proposition 7.11, $\left(f_{0}, g_{0}\right)=\left(\bigsqcup_{i \in \omega} T_{i} \searrow X_{i}, T_{0} \searrow B\right)$ is strongly consistent. Furthermore, we have $H_{\mathbf{I} v}\left(f_{0}\right) \sqsupseteq g_{0}$ since, for any $T \in \mathbf{I} T_{0}$, we obtain

$$
H_{\mathbf{I} v}\left(f_{0}\right)(T)=\mathbf{I} v\left(T,\left(\bigsqcup_{i \in \omega} T_{i} \searrow X_{i}\right)(T)\right) \sqsupseteq \mathbf{I} v\left(T, X_{0}\right) \sqsupseteq B \sqsupseteq g_{0}(T) .
$$

Since $f_{s}\left(\left\{t_{0}\right\}\right) \sqsupseteq f_{0}\left(\left\{t_{0}\right\}\right)=\left\{x_{0}\right\}$, Proposition 7.10 implies that $w_{f_{s}}=0$. Since $\mathbf{I} v$ preserves maximal elements, it follows from $g_{s}=H\left(\mathbf{I} v, f_{s}\right)$ that $w_{g_{s}}=0$ and $f_{s}$ and $g_{s}$ preserve maximal elements. Now, by Proposition 6.11, it follows that the induced maps $f_{s}, g_{s}$ : $T_{0} \rightarrow \mathbb{R}$ satisfy $f_{s}^{\prime}=g_{s}$ and hence $f_{s}^{\prime}(t)=v\left(t, f_{s}(t)\right)$ by the fix-point equation for $g_{s}$.

See Edalat et al. (2003) for an algorithmic framework for solving initial value problems based on this method. Since the first version of the current work became available, a simpler domain-theoretic approach to Picard's theorem for solving initial value problems has been developed in Edalat and Pattinson (2003).

\section{Implicit function theorem}

As explained in the Introduction, one of the main applications of the domain-theoretic model for differential calculus is to obtain piecewise smooth finitary approximations of curves and surfaces that are defined implicitly by, say, $f(\vec{x})=0$ where $f:[0,1]^{n} \rightarrow \mathbb{R}$ is a $C^{k}$ function of $n$ variables.

For functions of a single variable, which are the object of this paper, the implicit function theorem takes a very simple form: if $f \in C^{1}[0,1]$ and $f\left(x_{0}\right)=0$ with $f^{\prime}\left(x_{0}\right)>0$ for some $x_{0} \in(0,1)$, then $x_{0}$ is an isolated root of $f(x)=0$. Suppose now that $f$ and $f^{\prime}$ are computable maps in the sense of classical recursion theory (Pour-El and Richards 1988). Then $\mathbf{I} f$ and $\mathbf{I} f^{\prime}$ are computable (Edalat and Sünderhauf 1998) and can be approximated effectively by an increasing sequence of step functions $f=\bigsqcup_{i \in \omega} f_{i}$ and $g=\bigsqcup_{i \in \omega} g_{i}$. Since $\left(\mathbf{I} f, \mathbf{I} f^{\prime}\right) \in$ Cons, it follows that $\left(f_{i}, g_{i}\right) \in$ Cons for all $i \in \omega$. From $f^{\prime}\left(x_{0}\right)>0$, it follows that for some $i \in \omega$ we have $g_{i}\left(\left\{x_{0}\right\}\right)>0$ and thus the piecewise linear (or piecewise rational or semi-rational polynomial) functions $s\left(f_{i}, g_{i}\right)$ and $t\left(f_{i}, g_{i}\right)$ are both strictly increasing in a small neighbourhood of $x_{0}$. Therefore, they have computable roots $v_{i}$ and $w_{i}$ respectively with $x_{0} \in\left[w_{i}, v_{i}\right]$. In the limit we have $\left\{x_{0}\right\}=\bigcap_{i \in \omega}\left[w_{i}, v_{i}\right]$. This then gives us an algorithm for computing $x_{0}$ up to any desired accuracy. 


\section{Conclusion}

We have developed the two notions of the set of primitive maps and the derivative of a Scott continuous map and have shown that they are dually related in a similar way to the duality of differentiation and integration in the fundamental theorem of calculus. In the case of locally Lipschitz maps, the domain-theoretic derivative coincides with the Clarke's derivative. We have also constructed a domain-theoretic data type for $C^{1}$ functions in differential calculus, which extends the applications of domain theory and type theory to smooth mathematics. This domain also provides us with a model for the differential properties of piecewise $C^{1}$ functions, locally Lipschitz functions and, more generally, continuous functions. A domain-theoretic generalisation of Picard's theorem in this framework allows us to solve differential equations in such a way that at each stage of computation the approximation is bounded from below and above by two piecewise polynomial functions.

The domain construction for $C^{1}$ functions and some other results in this paper can be generalised to $C^{k}$ and $C^{\infty}$ functions. It can be shown that consistency of the basis elements for the domain of $C^{2}$ functions is decidable, but for $C^{k}$, with $k \geqslant 3$, the decidability of consistency is an open question.

We are also developing and implementing the algorithmic framework for solving differential equations using the domain-theoretic version of Picard's theorem as formulated algorithmically in the follow-up papers Edalat et al. (2003) and Edalat and Pattinson (2003).

Since the first version of this paper was produced, the domain-theoretic framework for differential calculus has been extended to functions of several variables in Edalat et al. (2004). This extension will allow us to develop domain-theoretic effective versions of the inverse and implicit functions theorem, which are the building blocks of multi-dimensional differential calculus with, in particular, applications to the approximation of curves and surfaces.

\section{Acknowledgements}

We thank John Howroyd, Marko Krznaric, Ali Khanban, Jimmie Lawson and Dirk Pattinson for discussions or comments. This work has been supported by EPSRC.

\section{References}

Abramsky, S. and Jung, A. (1994) Domain theory. In: Abramsky, S., Gabbay, D. M. and Maibaum, T. S. E. (eds.) Handbook of Logic in Computer Science 3, Clarendon Press.

Amadio, R.M. and Curien, P.-L. (1998) Domains and Lambda-Calculi, Cambridge Tracts in Theoretical Computer Science.

Aubin, J. P. and Cellina, A. (1984) Differential Inclusions, Springer.

Clarke, F. H. (1983) Optimization and Nonsmooth Analysis, Wiley.

Clarke, F. H., Ledyaev, Y.S., Stern, R. J. and Wolenski, P. R. (1998) Nonsmooth Analysis and Control Theory, Springer.

Edalat, A. (1995a) Domain theory and integration. Theoretical Computer Science 151 163-193. 
Edalat, A. (1995b) Dynamical systems, measures and fractals via domain theory. Information and Computation 120 (1) 32-48.

Edalat, A. (1997) Domains for computation in mathematics, physics and exact real arithmetic. Bulletin of Symbolic Logic 3 (4) 401-452.

Edalat, A. and Escardó, M. (2000) Integration in real PCF. Information and Computation 160 $128-166$.

Edalat, A. and Heckmann, R. (1998) A computational model for metric spaces. Theoretical Computer Science 193 (1-2) 53-73.

Edalat, A., Krznarić, M. and Lieutier, A. (2003) Domain-theoretic solution of differential equations (scalar fields). In: Proceedings of MFPS XIX. Electronic Notes in Theoretical Computer Science 83 (Full paper available from www.doc.ic.ac.uk/ ae/papers/scalar.ps.)

Edalat, A. and Lieutier, A. (2002) Foundation of a computable solid modelling. Theoretical Computer Science 284 (2) 319-345.

Edalat, A., Lieutier, A. and Pattinson, D. (2004) Domain theory and multi-variable calculus. Available from www.doc.ic.ac.uk/ ae/papers/multi.ps.

Edalat, A. and Pattinson, D. (2003) Initial value problems in domain theory. In: Proceedings of Computability and Complexity in Analysis 2003, (CCA'03). (Full paper available from www.doc.ic.ac.uk/ ${ }^{\sim a e} /$ papers/picard.icalp.ps.)

Edalat, A. and Potts, P. J. (1997) A new representation for exact real numbers. In: Proceedings of Mathematical Foundations of Programming Semantics 13. Electronic Notes in Theoretical Computer Science 6. Available from www.elsevier.nl/locate/entcs/volume6.html.

Edalat, A. and Sünderhauf, P. (1998) A domain theoretic approach to computability on the real line. Theoretical Computer Science 210 73-98.

Escardó, M.H. (1996) PCF extended with real numbers. Theoretical Computer Science 162 (1) $79-115$.

Gierz, G., Hofmann, K.H., Keimel, K., Lawson, J.D., Mislove, M. and Scott, D.S. (1980) A Compendium of Continuous Lattices, Springer Verlag.

Hewitt, E. and Stromberg, K. (1975) Real and abstract analysis: a modern treatment of the theory of functions of a real variable, Graduate texts in mathematics 25, Springer.

Hu, C.-Y., Maekawa, T., Sherbrooke, E. C. and Patrikalakis, N. M. (1996) Robust interval algorithm for curve intersections. Computer-Aided Design 28 (6-7) 495-506.

Kolmogorov, A. N. and Fomin, S. V. (1975) Introductory Real Analysis, Dover.

Lawson, J. (1997) Spaces of maximal points. Mathematical Structures in Computer Science 7 (5) $543-555$.

Martin, K. (2000) A Foundation for Computation, Ph.D. thesis, University of Tulane.

Moore, R. (1966) Interval Analysis, Prentice-Hall, Englewood Cliffs.

Plotkin, G. D. (1981) Post-graduate lecture notes in advanced domain theory (incorporating the 'Pisa Notes'). Department of Computer Science, University of Edinburgh. (Available in IATEX.)

Pour-El, M. B. and Richards, J. I. (1988) Computability in Analysis and Physics, Springer-Verlag.

Sakkalis, Y., Shen, G. and Patrikalakis, N. M. D. (2001) Topological and geometric properties of interval solid models. Graphical Models 63 163-175.

Smyth, M. B. (1977) Effectively given domains. Theoretical Computer Science 5 257-274.

Stoltenberg-Hansen, V., Lindström, I. and Griffor, E. R. (1994) Mathematical Theory of Domains, Cambridge Tracts in Theoretical Computer Science 22, Cambridge University Press.

Weihrauch, K. (2000) Computable Analysis (An Introduction), Springer. 